\title{
The circumstellar environment of high-mass protostellar objects
}

\section{IV. $\mathrm{C}^{17} \mathrm{O}$ observations and depletion ${ }^{\star}$}

\author{
H. S. Thomas and G. A. Fuller \\ Jodrell Bank Centre for Astrophysics, Alan Turing Building, The University of Manchester, Manchester M13 9PL, UK \\ e-mail: H.Thomas@postgrad.manchester.ac.uk
}

Received 3 October 2006 / Accepted 20 November 2007

\begin{abstract}
Aims. The presence of depletion (freeze-out) of $\mathrm{CO}$ around low-mass protostars is well established. Here we observe 84 candidate young high-mass sources in the rare isotopologues $\mathrm{C}^{17} \mathrm{O}$ and $\mathrm{C}^{18} \mathrm{O}$ to investigate whether there is evidence for depletion towards these objects.

Methods. Observations of the $J=2 \rightarrow 1$ transitions of $\mathrm{C}^{18} \mathrm{O}$ and $\mathrm{C}^{17} \mathrm{O}$ are used to derive the column densities of gas towards the sources and these are compared with those derived from submillimetre continuum observations. The derived fractional abundance suggests that the $\mathrm{CO}$ species show a range of degrees of depletion towards the objects. We then use the radiative transfer code RATRAN to model a selection of the sources to confirm that the spread of abundances is not a result of assumptions made when calculating the column densities.

Results. We find a range of abundances of $\mathrm{C}^{17} \mathrm{O}$ that cannot be accounted for by global variations in either the temperature or dust properties and so must reflect source to source variations. The most likely explanation is that different sources show different degrees of depletion of the CO. Comparison of the $\mathrm{C}^{17} \mathrm{O}$ linewidths of our sources with those of CS presented by other authors reveal a division of the sources into two groups. Sources with a CS linewidth $>3 \mathrm{~km} \mathrm{~s}^{-1}$ have low abundances of $\mathrm{C}^{17} \mathrm{O}$ while sources with narrower CS lines have typically higher $\mathrm{C}^{17} \mathrm{O}$ abundances. We suggest that this represents an evolutionary trend. Depletion towards these objects shows that the gas remains cold and dense for long enough for the trace species to deplete. The range of depletion measured suggests that these objects have lifetimes of $2-4 \times 10^{5}$ years.
\end{abstract}

Key words. ISM: molecules - line: profiles - stars: abundances - stars: evolution - stars: formation

\section{Introduction}

Many questions regarding the formation mechanism of highmass stars remain unanswered. Young high-mass stars form within massive cores and display features common to low-mass star formation such as outflows and in some cases disks (e.g. Shepherd 2005; Cesaroni et al. 2007). However, the different stages through which a core forming a high-mass star evolves remains unclear.

In the cold, dense conditions within cores prior to their collapse, trace molecules freeze-out onto the dust grains forming icy mantles. This process reduces the abundances of species in the gas phase until the grains are heated, desorbing the molecules off the grains and returning them back into the gas. This freezeout and evaporation cycle is potentially an important indicator of the age of the material in star forming cores and how the material has evolved.

The transitions of the two rare isotopologues of $\mathrm{CO}, \mathrm{C}^{18} \mathrm{O}$ and $\mathrm{C}^{17} \mathrm{O}$ which have abundances with respect to $\mathrm{H}_{2}$ of $\sim 2 \times$ $10^{-7}$ and $\sim 5 \times 10^{-8}$ respectively (Frerking et al. 1982), are often employed to probe the inner regions of dense cores (Tafalla et al. 2002; Caselli et al. 1999; Redman et al. 2002) as their transitions are typically optically thin, especially those of $\mathrm{C}^{17} \mathrm{O}$. A number

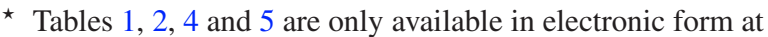
http://www. aanda. org of studies of various, but mainly low-mass, star forming regions using these species have provided evidence for depletion of $\mathrm{CO}$ towards the centres of cores (Caselli et al. 1999; Kramer et al. 1999; Tafalla et al. 2002; Willacy et al. 1998; Savva et al. 2003) with the degree of depletion varying from factors of a few to over an order of magnitude compared to the canonical $\mathrm{CO}$ abundance.

In this paper, we present new observations of candidate highmass star forming regions in $\mathrm{C}^{17} \mathrm{O}$ and $\mathrm{C}^{18} \mathrm{O}$ made in order to look for evidence of depletion in these regions.

\section{Observations of $C^{17} \mathrm{O}$ and $\mathrm{C}^{18} \mathrm{O}$}

Sridharan et al. (2002, hereafter SBSMW) searched the IRAS catalogue and identified 69 point sources which represent potentially massive, deeply embedded protostars in the Galactic plane. These sources have been studied extensively by Beuther et al. (2002a,b) and have been mapped with SCUBA, the James Clerk Maxwell Telescope (JCMT) bolometer array, at $850 \mu \mathrm{m}$ and $450 \mu \mathrm{m}$ by Williams et al. (2004, hereafter WFS04) as well as being surveyed for evidence of infall by Fuller et al. (2005). In many cases the submillimetre and millimetre continuum maps show more than a single peak indicating multiple sites of potential star formation. In total $112850 \mu \mathrm{m}$ peaks were identified, of which we have observed 84 in $\mathrm{C}^{17} \mathrm{O}$.

We observed the $J=2 \rightarrow 1$ rotational transitions of $\mathrm{C}^{18} \mathrm{O}$ $(219.560 \mathrm{GHz})$ and $\mathrm{C}^{17} \mathrm{O}(224.714 \mathrm{GHz})$ towards 31 sources 
during May 2004 at the $\mathrm{JCMT}^{1}$ in Hawaii as part of observing program M04AU47. We used the RxA3 receiver with the DAS autocorrelator with channels of $78 \mathrm{kHz}$ in position switching mode using a reference position of $\left(+600^{\prime \prime},+600^{\prime \prime}\right)$. The reference positions were chosen to be free of $\mathrm{C}^{18} \mathrm{O}$ emission having been checked and used for previous molecular observations. The pointing was checked every couple of hours. The typical zenith opacity at $225 \mathrm{GHz}$ was between 0.15 and 0.4 and we achieved a typical rms of $\lesssim 0.25 \mathrm{~K}$. The data were baseline subtracted using the SPECX package following normal routines.

As we are primarily interested in deriving column densities, in further observations we chose to concentrate on the $\mathrm{C}^{17} \mathrm{O}$ line which has lower optical depth. A further 53 sources were observed in $\mathrm{C}^{17} \mathrm{O} J=2 \rightarrow 1$ in July and August 2005 (in observing program M05BU46). In addition we observed a selection of these sources in the $J=3 \rightarrow 2$ transition of $\mathrm{C}^{17} \mathrm{O}(337.061 \mathrm{GHz})$ with the RxB3 receiver. This time we achieved a typical rms of $\$ 0.15 \mathrm{~K}$. These data were processed in the same way as the earlier data.

As the telescope beam is not perfectly coupled to the source it is necessary to apply a correction factor to convert antenna temperature into a main beam temperature where

$T_{\mathrm{mb}}=\frac{T_{\mathrm{A}}^{*}}{\eta_{\mathrm{mb}}}$.

At the JCMT the main beam efficiencies are 0.69 at $220 \mathrm{GHz}$ with a beam size of $20^{\prime \prime}$ and 0.63 at $337 \mathrm{GHz}$ with a beam size of $13^{\prime \prime}$. The positions and dates of observations for all the sources are given in Table 1.

\section{Analysis}

\subsection{Line profiles}

The line parameters were initially measured using SPECX by fitting Gaussian line-profiles to derive the source velocity $\left(v_{\mathrm{lsr}}\right)$, line width $(\Delta v)$, peak line flux $\left(T_{\mathrm{A}}^{*}\right)$ and integrated line intensities.

All the $\mathrm{C}^{18} \mathrm{O}$ data were fitted with a single Gaussian. These fits produced typical peak temperatures of $T_{\mathrm{A}}^{*} \sim 2.5-7.5 \mathrm{~K}$, and linewidths of $\Delta v \sim 1.5-3.5 \mathrm{~km} \mathrm{~s}^{-1}$. However, examination reveals that a significant fraction of these $\mathrm{C}^{18} \mathrm{O}$ data $(\sim 65 \%)$ are distinctly better fit, with smaller residuals, by the sum of two different velocity components (Fig. 1). The results of two components fits are also given in Table 2 . Those sources with multiple components can be divided into two categories; those where the 2 components are of approximately equal width, but are offset in velocity, and those where there is a definite separation into a broad and a narrow component, with the broad component possibly being a related to the outflow from the source. In this latter case the typical linewidths of the broad and narrow components are $\Delta v_{\text {br }} \geq 3 \mathrm{~km} \mathrm{~s}^{-1}$ and $\Delta v_{\mathrm{n}} \leq 2 \mathrm{~km} \mathrm{~s}^{-1}$.

The integrated intensities for $\mathrm{C}^{17} \mathrm{O}$ have been calculated from a Gaussian fit using SPECX. However unlike the $\mathrm{C}^{18} \mathrm{O}$, the transitions of $\mathrm{C}^{17} \mathrm{O}$ have hyperfine structure. Simple Gaussian fits to the $\mathrm{C}^{17} \mathrm{O}$ data therefore overestimate the intrinsic velocity dispersion. The $\mathrm{C}^{17} \mathrm{O}$ line parameters were therefore fitted with the known hyperfine structure (hfs) of the respective transitions using METHOD HFS in the CLASS package assuming all components have equal excitation temperatures. For the

1 The James Clerk Maxwell Telescope is operated by The Joint Astronomy Centre on behalf of the Particle Physics and Astronomy Research Council of the United Kingdom, the Netherlands Organisation for Scientific Research, and the National Research Council of Canada.
$J=2 \rightarrow 1$ transition there are 9 hyperfine components, while there are 14 for the $J=3 \rightarrow 2$ transition. The line parameters, the peak intensity, the $v_{\text {lsr }}$, the linewidth and the integrated intensity are given in Tables 3 and 4. The optical depths calculated from the hfs fits imply these sources are optically thin in $\mathrm{C}^{17} \mathrm{O}$ with $\tau \sim 0.1$.

\subsection{Ratio of integrated intensities}

The ratio of $\mathrm{C}^{18} \mathrm{O}$ to $\mathrm{C}^{17} \mathrm{O}$ line intensities can constrain the optical depth of the line if the abundance ratio is known (Ladd et al. 1998). Penzias conducted a survey of 15 massive star forming regions lying in the galactic disk (Penzias 1981) and derived a value of $R=\left[{ }^{18} \mathrm{O}\right] /\left[{ }^{17} \mathrm{O}\right]=3.65 \pm 0.15$ and found no gradient based on galactocentric distance. Further work by different authors have produced values, assuming that $\left[\mathrm{C}^{18} \mathrm{O}\right] /\left[\mathrm{C}^{17} \mathrm{O}\right]=$ $\left[{ }^{18} \mathrm{O}\right] /\left[{ }^{17} \mathrm{O}\right]$, ranging from $R=2.9 \pm 1.2$ (Sheffer et al. 2002) to $4.15 \pm 0.52$ (Bensch et al. 2001). More recently Ladd (2004) conducted a survey of 648 lines of sight towards 5 star forming regions in the Taurus molecular cloud and combining the results for the individual regions concluded that $R=4.0 \pm 0.5$, a value consistent with an analysis of other clouds by Wouterloot et al. (2005).

A comparison of our measured $\mathrm{C}^{17} \mathrm{O}$ and $\mathrm{C}^{18} \mathrm{O}$ integrated intensities is shown in Fig. 2. Assuming equal excitation temperatures and beam filling factors for the two species, if the emission is optically thin one would expect the ratio of the integrated line intensity of the two species to equal the abundance ratio. A standard value of 3.65 for the abundance ratio of $\mathrm{C}^{18} \mathrm{O}$ to $\mathrm{C}^{17} \mathrm{O}$ is indicated by the solid line. Of the sources, 5 lie approximately on the line implying that for the $\mathrm{C}^{18} \mathrm{O}$ and $\mathrm{C}^{17} \mathrm{O}$ lines are optically thin. The absence of any sources above the line is consistent with both the abundance ratio values of Penzias (3.65) and Ladd (4.0), but not higher values. As so many of our sources lie close to this line it suggests that the value of 3.65 is the upper limit for this ratio. However, the majority of our sources lie below this value suggesting that the $\mathrm{C}^{18} \mathrm{O}$ emission is not optically thin. The figure also shows the expected ratio of integrated intensities for optical depth 2 in the $\mathrm{C}^{18} \mathrm{O}$ transition. Due to the overlap of the hyperfine components of the $\mathrm{C}^{17} \mathrm{O}$ the optical depth at the line peak depends on the velocity dispersion. The line on the figure has been calculated for a FWHM velocity of $3 \mathrm{~km} \mathrm{~s}^{-1}$ (and a $\mathrm{C}^{18} \mathrm{O}$ to $\mathrm{C}^{17} \mathrm{O}$ abundance ratio of 3.65) giving a corresponding $\mathrm{C}^{17} \mathrm{O}$ peak optical depth of 0.5 . If the FWHM velocity was $1 \mathrm{~km} \mathrm{~s}^{-1}$, the $\mathrm{C}^{17} \mathrm{O}$ optical depth would be reduced by a factor of 0.67 , whereas if the the FWHM velocity was $5 \mathrm{~km} \mathrm{~s}^{-1}$, the optical depth would be increased by a factor of 1.05 . The figure shows that the $\mathrm{C}^{17} \mathrm{O}$ emission is not highly optically thick towards these sources, a result consistent with optical depth implied by the fit to the hyperfine structure of the observed lines (Sect. 3.1). The figure also shows that there is no correlation between line strength and optical depth.

\subsection{Column densities}

Since the $\mathrm{C}^{17} \mathrm{O}$ emission appears to not be optically thick, we calculate the column densities assuming that the $\mathrm{C}^{17} \mathrm{O}$ is optically thin. This is consistent with the analysis of $\mathrm{C}^{17} \mathrm{O}$ towards similar objects by both van der Tak et al. (2000) and Fontani et al. (2006). 
Table 3. Observed line parameters. The columns give the peak intensity, velocity, linewidth and integrated intensity for $\mathrm{C}^{18} \mathrm{O}$ and $\mathrm{C}^{17} \mathrm{O}$ respectively. For $\mathrm{C}^{17} \mathrm{O}$ the first three parameters are derived from hyperfine fitting of the data using CLASS. The integrated intensity was obtained using a single Gaussian fit.

\begin{tabular}{|c|c|c|c|c|c|c|c|c|}
\hline \multirow[b]{2}{*}{ WFS } & \multicolumn{4}{|c|}{$\mathrm{C}^{18} \mathrm{O} J=2 \rightarrow 1$} & \multicolumn{3}{|c|}{$\mathrm{C}^{17} \mathrm{O} J=2 \rightarrow 1$} & \multirow[b]{2}{*}{$\int_{\left(\mathrm{K} \mathrm{km} \mathrm{s}^{-1}\right)} T_{\mathrm{mb}} \mathrm{d} v$} \\
\hline & $\begin{array}{c}\text { Peak } T_{\mathrm{mb}} \\
(\mathrm{K})\end{array}$ & $\begin{array}{c}v_{\text {lss }} \\
\left(\mathrm{km} \mathrm{s}^{-1}\right)\end{array}$ & $\begin{array}{c}\Delta v \\
\left(\mathrm{~km} \mathrm{~s}^{-1}\right)\end{array}$ & $\int_{\left(\mathrm{K} \mathrm{km} \mathrm{s}{ }^{-1}\right)} T_{\mathrm{mb}} \mathrm{d} v$ & $\begin{array}{c}\text { Peak } T_{\mathrm{mb}} \\
(\mathrm{K})\end{array}$ & $\begin{array}{c}v_{\text {lsr }} \\
\left(\mathrm{km} \mathrm{s}^{-1}\right)\end{array}$ & $\begin{array}{c}\Delta v \\
\left(\mathrm{~km} \mathrm{~s}^{-1}\right)\end{array}$ & \\
\hline 14 & 9.77 & 33.26 & 2.42 & 25.61 & 2.59 & 33.22 & 2.45 & 7.75 \\
\hline 16 & 10.54 & 59.22 & 3.36 & 37.38 & 3.97 & 59.19 & 3.00 & 14.03 \\
\hline 17 & 10.74 & 45.08 & 2.72 & 32.74 & 3.45 & 45.09 & 2.67 & 11.65 \\
\hline 20 & 3.30 & 34.39 & 2.82 & 9.80 & 1.26 & 34.28 & 2.71 & 4.19 \\
\hline 21 & 5.19 & 34.39 & 2.25 & 12.74 & 1.75 & 34.35 & 2.29 & 5.22 \\
\hline 22 & 8.99 & 84.51 & 2.23 & 21.42 & 3.09 & 84.48 & 2.01 & 8.01 \\
\hline 25 & 7.17 & 77.85 & 2.47 & 19.64 & 2.52 & 77.78 & 2.25 & 7.62 \\
\hline 29 & 5.48 & 58.94 & 2.69 & 15.22 & 2.67 & 59.24 & 2.16 & 7.59 \\
\hline 30 & 4.06 & 95.69 & 2.44 & 13.01 & 0.96 & 95.75 & 2.87 & 3.70 \\
\hline 34 & 8.09 & 22.83 & 3.39 & 30.06 & 2.62 & 22.96 & 3.41 & 10.97 \\
\hline 36 & 4.67 & 15.79 & 2.64 & 16.12 & 1.99 & 15.93 & 2.58 & 6.39 \\
\hline 39 & 5.13 & 96.07 & 2.64 & 17.28 & 1.91 & 96.04 & 2.63 & 6.46 \\
\hline 51 & 7.75 & 84.29 & 1.85 & 14.84 & 2.32 & 84.33 & 1.75 & 5.16 \\
\hline 78 & 4.99 & 21.72 & 2.83 & 15.12 & 1.32 & 21.80 & 2.47 & 4.22 \\
\hline 79 & 6.01 & 22.53 & 2.85 & 19.07 & 1.74 & 22.54 & 2.34 & 6.10 \\
\hline 85 & - & - & - & - & 1.41 & 11.20 & 2.40 & 4.45 \\
\hline 87 & - & - & - & - & 2.64 & 5.58 & 1.26 & 5.04 \\
\hline 88 & - & - & - & - & 4.25 & 5.83 & 0.93 & 6.43 \\
\hline 90 & 5.43 & -3.64 & 2.83 & 17.29 & 1.46 & -3.67 & 2.92 & 5.54 \\
\hline 91 & 10.38 & -1.89 & 1.26 & 14.01 & 3.13 & -1.85 & 1.20 & 5.78 \\
\hline 95 & - & - & - & - & 0.94 & 8.52 & 2.33 & 2.80 \\
\hline 96 & - & - & - & - & 1.87 & -3.19 & 2.01 & 4.89 \\
\hline 97 & - & - & - & - & 1.33 & -2.19 & 2.77 & 4.36 \\
\hline 99 & 6.01 & 11.16 & 2.10 & 13.46 & 1.80 & 11.12 & 1.80 & 4.23 \\
\hline 100 & 4.94 & 11.40 & 2.85 & 14.12 & 1.54 & 11.40 & 2.60 & 4.81 \\
\hline 107 & 4.09 & -45.85 & 2.25 & 10.38 & 1.26 & -45.86 & 1.85 & 3.01 \\
\hline 108 & 5.10 & -53.18 & 2.79 & 16.48 & 1.52 & -53.11 & 2.82 & 5.88 \\
\hline 109 & 4.36 & -44.29 & 2.74 & 13.47 & 1.29 & -44.38 & 2.33 & 4.00 \\
\hline 110 & 3.91 & -54.71 & 2.31 & 10.09 & 1.22 & -54.73 & 2.39 & 3.62 \\
\hline 111 & 10.17 & -17.94 & 1.46 & 15.81 & 3.29 & -17.88 & 1.26 & 6.28 \\
\hline 112 & 6.68 & -18.45 & 1.84 & 12.51 & 1.87 & -18.62 & 1.55 & 4.01 \\
\hline
\end{tabular}

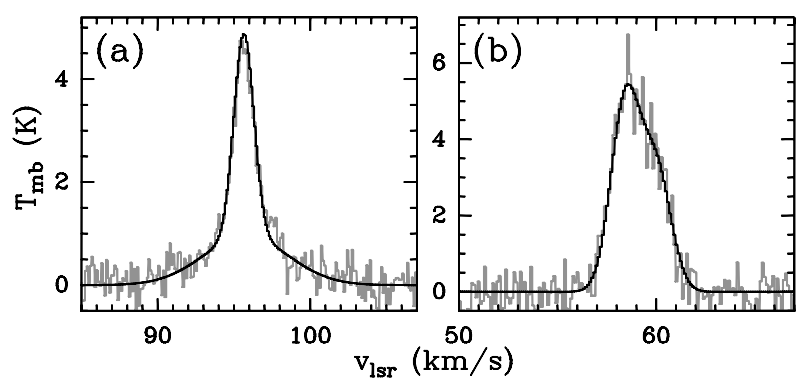

Fig. 1. Example spectra of $\mathrm{C}^{18} \mathrm{O}$ line profiles for a) WFS30 and b) WFS 29 which are best fit by 2 Gaussian components shown as the smooth curve over each line.

To calculate the $\mathrm{CO}$ column densities we used the following general expression assuming LTE and optically thin emission;

$N_{\text {tot }}=\frac{3 k}{8 \pi^{3} v \mu^{2} g_{\mathrm{u}}} \frac{Q\left(T_{\mathrm{ex}}\right)}{\exp \left(-E_{\mathrm{u}} / k T_{\mathrm{ex}}\right)} \int T_{\mathrm{mb}} \mathrm{d} v$.

Here $g_{\mathrm{u}}$ is the statistical weight, $Q(T)$ is the partition function and $\mu$ is the electric dipole moment for the molecule. Expressing the integrated intensity in $\mathrm{K} \mathrm{km} \mathrm{s}^{-1}$, the dipole moment in Debye $(\mu=0.11 \mathrm{D}$ for $\mathrm{CO})$ and the frequency in $\mathrm{GHz}$, this reduces to

$N_{\text {tot }}=1.67 \times 10^{14} \frac{Q\left(T_{\text {ex }}\right)}{\mu^{2}(\text { Debye }) v(\mathrm{GHz}) g_{\mathrm{u}}} \exp \left(\frac{E_{\mathrm{u}}}{T_{\mathrm{ex}}}\right) \int T_{\mathrm{mb}} \mathrm{d} v$.

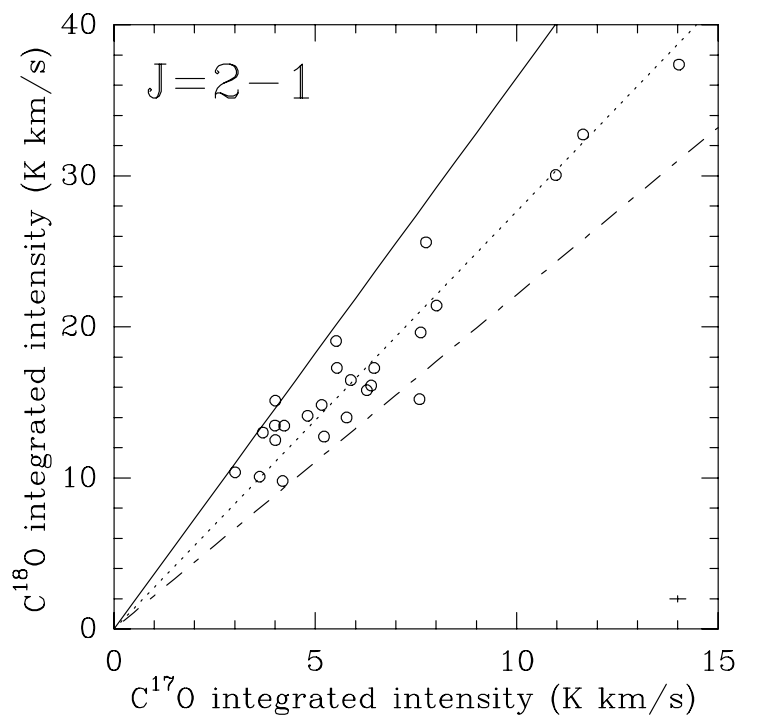

Fig. 2. Comparison of the integrated intensities of $\mathrm{C}^{18} \mathrm{O}$ and $\mathrm{C}^{17} \mathrm{O}$. The solid line denotes an abundance ratio of $\mathrm{C}^{17} \mathrm{O}$ to $\mathrm{C}^{18} \mathrm{O}$ of 3.65 , the expected value if both lines are optically thin (Penzias 1981; Ladd et al. 1998). The dotted line indicates the best-fit curve of 2.8. The dashed line indicates an optical depth of 2 associated with $\mathrm{C}^{18} \mathrm{O}$.

This method assumes a constant excitation temperature $\left(T_{\mathrm{ex}}\right)$ along the line of sight which is a free parameter in the analysis. 
For all their observed sources, SBSMW also estimated the temperature of the cold component of the dust; the mean over the whole sample was $45 \mathrm{~K}$. SBSMW also measured the gas temperature towards many of the sources observed here using $\mathrm{NH}_{3}$. Over their entire sample SBSMW find a mean temperature of $19 \mathrm{~K}$ and for all but two of the sources observed here they found temperatures of $<20 \mathrm{~K}$. To estimate the $\mathrm{C}^{17} \mathrm{O}$ column density we have adopted $30 \mathrm{~K}$, a compromise value intermediate between cold dust and $\mathrm{NH}_{3}$ temperatures. The column density is of course sensitive to the assumed excitation temperature and is at a minimum at $T_{\mathrm{ex}}=17 \mathrm{~K}$. At $10 \mathrm{~K}$ and $30 \mathrm{~K}$ the column densities are approximately equal and about $16 \%$ higher than at the minimum.

For comparison with the observations of the dust towards the sources it was necessary to re-convolve the $850 \mu \mathrm{m}$ data to a beamsize to match that of the $\mathrm{CO}$ beam. We then calculated the mass in the beam using the expression

$M_{\text {(beam })}=\frac{S_{v(\text { beam })} d^{2}}{\kappa_{v} B_{v}\left(T_{\text {dust }}\right)}$

where we take the value for the opacity from WFS04 as $\kappa_{850}=$ $1.54 \times 10^{-2} \mathrm{~cm}^{2} \mathrm{~g}^{-1}$ and the dust temperature $\left(T_{\text {dust }}\right)$ as the cold dust component given in SBSMW. We then derive the beamaveraged hydrogen column density from the submillimetre mass using the relationship from Hildebrand (1983) given as

$M_{\text {(beam) }}=\left[\pi \theta_{\frac{1}{2}}^{2} d^{2}\right] N\left(H+H_{2}\right) m_{\mathrm{H}} \mu$

where $m_{\mathrm{H}}$ is the mass of a hydrogen atom and $\mu$ is the ratio of total gas mass to hydrogen mass and $\theta_{\frac{1}{2}}$ is the beam radius. In both steps the distance to the source, $d$, is taken to be the near-distance if there is an ambiguity (SBSMW). The results for $N\left(\mathrm{C}^{17} \mathrm{O}\right)$ are given along with the hydrogen column density derived from the dust continuum emission in Table 5.

Figure 3 shows the column density $\mathrm{C}^{17} \mathrm{O}$ plotted against that calculated for $\mathrm{H}_{2}$ from the dust continuum emission. Also shown are lines of constant $\mathrm{CO}$ abundance chosen to constrain the data. It is immediately evident that this graph shows a large scatter in the abundances. The $\mathrm{C}^{17} \mathrm{O}$ data are constrained by abundances of $\sim 1 \times 10^{-7}$ and $\sim 7 \times 10^{-9}$, a factor of approximately fourteen spread in the abundance. Taking the standard $\left[\mathrm{C}^{17} \mathrm{O}\right] /\left[\mathrm{H}_{2}\right]$ abundance to be $4.7 \times 10^{-8}$ (Frerking et al. 1982), we would expect our sources to lie either on this line or below it in the case of $\mathrm{CO}$ depletion, yet it is clear that this value does not represent the upper limit for our sources and a significant fraction lie up to a factor of 2 above this line.

To explore the reliability of the derived $\mathrm{C}^{17} \mathrm{O}$ abundances, we have also run detailed models of the emission expected from these sources. These models allow us to investigate the possible consequences of some of the assumptions made when calculating the column densities, particularly the assumed excitation temperature and dust temperature, together with the role of density gradients within the beam.

\section{Modelling}

To model the emission of $\mathrm{C}^{17} \mathrm{O}$ and $\mathrm{C}^{18} \mathrm{O}$ we used the $1 \mathrm{D}$ radiative transfer code RATRAN developed by Hogerheijde \& van der Tak (2000). RATRAN utilises the Monte Carlo method to calculate the radiative transfer of molecular lines through a dusty shell.

Williams et al. (2005, hereafter WFS05) modelled the $850 \mu \mathrm{m}$ emission for a selection of the sources observed by

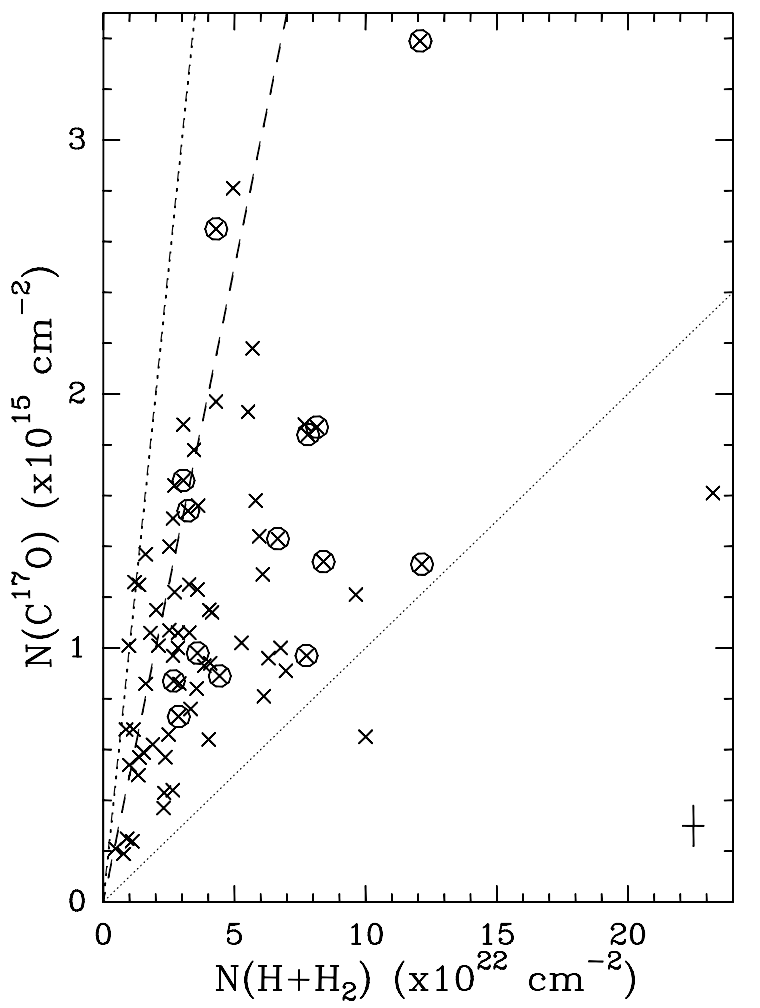

Fig. 3. Comparison of the column density of $\mathrm{C}^{17} \mathrm{O}$ with the $\mathrm{H}_{2}+\mathrm{H}$ column density derived from the dust continuum emission. Typical uncertainties are shown in the lower right corner of the panel. The dashed lines represent lines of constant abundance: Short dashed $=5 \times 10^{-7}$, dot-dashed $=1 \times 10^{-7}$, long dashed $=5 \times 10^{-8}$ and dotted $=1 \times$ $10^{-8}$. The circled sources indicate those which have been modelled (see Sect. 4).

WFS04 using the 1D radiative transfer code DUSTY (Ivezić et al. 1999) in order to determine the physical parameters of the envelopes around the central embedded sources. Of those sources for which we have both $\mathrm{C}^{17} \mathrm{O}$ and $\mathrm{C}^{18} \mathrm{O}$ data, 14 were successfully modelled by WFS05. We have utilised the parameters of the best-fit models from WFS05 to generate the input for RATRAN.

WFS05 identified certain sources as asymmetric and used radial slices to explore the structure in various directions, as a result four of our 14 sources actually have multiple models in WFS05. For these sources we correspondingly generated multiple models for RATRAN. These are indicated by alphabetical suffixes to the source names.

As RATRAN is a 1D code it models the sources as spherically symmetric and hence neglects any openings or elongations due to outflows or winds. The model assumes a dust-free cavity surrounding the star from radius $r=0$ to $r=r_{1}$. Between $r_{1}$ and $r_{2}$ lies the envelope to be modelled. We divided the radial distance between $r_{1}$ and $r_{2}$ into 30 logarithmically spaced shells to give increased resolution towards the inner region of the shell where the temperature and density profiles change most rapidly. Thirty shells were used corresponding to the number used by van der Tak et al. (2000, hereafter VVEB) in their RATRAN modelling of massive young stars. We also ran a number of identical models using both 15 and 60 shells and found a negligible effect on the output compared to the 30 shell models.

To determine the input for RATRAN we used the following parameters derived in WFS05: power-law index $(\alpha)$ of the envelope density profile $n(r)$ where $n \propto r^{-\alpha}$, the temperature at 
Table 6. Parameters used in the detailed modelling of the sources. Where $r_{1}$ is the inner radius of the modelled envelope, $r_{2}$ is the outer radius and $\alpha$ is the power-law index of the number density profile $n(r)$ where $n \propto r^{-\alpha}$. Column 5 gives the hydrogen number density at $r_{1}$.

\begin{tabular}{ccccccc}
\hline \hline WFS & $r_{1}$ & $r_{2}$ & $\alpha$ & $n\left(r_{1}\right)$ & $\Delta v\left(\mathrm{C}^{17} \mathrm{O}\right)$ & $\Delta v\left(\mathrm{C}^{18} \mathrm{O}\right)$ \\
\cline { 2 - 7 } & $10^{16} \mathrm{~cm}$ & $10^{18} \mathrm{~cm}$ & & $10^{6} \mathrm{~cm}^{-3}$ & $\mathrm{~km} \mathrm{~s}^{-1}$ & $\mathrm{~km} \mathrm{~s}^{-1}$ \\
\hline 14 & 3.02 & 1.51 & 1.5 & 3.09 & 2.84 & 2.21 \\
16 & 4.06 & 4.06 & 2.0 & 8.74 & 3.35 & 2.95 \\
25 & 2.69 & 5.39 & 1.5 & 1.33 & 2.64 & 2.20 \\
29 & 2.27 & 1.59 & 1.0 & 0.48 & 2.59 & 2.17 \\
30 & 0.23 & 1.84 & 1.0 & 4.41 & 2.37 & 2.27 \\
$34 \mathrm{a}$ & 1.84 & 0.18 & 1.0 & 32.97 & 3.71 & 3.30 \\
$34 \mathrm{~b}$ & 0.63 & 5.04 & 1.5 & 2.19 & 3.71 & 3.30 \\
36 & 0.45 & 0.90 & 1.0 & 0.83 & 2.94 & 2.32 \\
79 & 2.41 & 1.21 & 1.5 & 6.78 & 2.71 & 2.33 \\
$90 \mathrm{a}$ & 1.78 & 1.25 & 1.5 & 3.44 & 3.27 & 2.87 \\
$90 \mathrm{~b}$ & 1.83 & 1.83 & 2.0 & 12.74 & 3.27 & 2.87 \\
107 & 3.50 & 2.45 & 0.5 & 0.05 & 2.24 & 1.59 \\
$108 \mathrm{a}$ & 0.64 & 2.56 & 1.5 & 8.35 & 3.25 & 2.83 \\
$108 \mathrm{~b}$ & 0.22 & 3.52 & 1.0 & 1.07 & 3.25 & 2.83 \\
109 & 0.23 & 3.68 & 1.5 & 33.80 & 2.54 & 2.00 \\
$110 \mathrm{a}$ & 0.64 & 2.56 & 1.5 & 5.19 & 2.82 & 2.09 \\
$110 \mathrm{~b}$ & 1.70 & 3.40 & 1.5 & 0.80 & 2.82 & 2.09 \\
$110 \mathrm{c}$ & 1.83 & 1.83 & 2.0 & 16.68 & 2.82 & 2.09 \\
\hline
\end{tabular}

the inner boundary $T_{1}$, the scale of the envelope defined by the inner radius, $r_{1}$ and $Y$, where $Y$ is the ratio between $r_{2}$ and $r_{1}$. The outer radius can then be calculated from $r_{2}=Y r_{1}$.

We generated the density profile for each model by combining the power-law index $(\alpha)$ of the envelope density profile given in WFS05 with the density at $r_{1}$ which we calculate from the $850 \mu \mathrm{m}$ mass and volume of the envelope (WFS04). As the dust temperature profile is not given in WFS05 we regenerated the WFS05 models using DUSTY to determine the individual temperature profile for each model. This profile was then interpolated to the radii of the 30 shells used to trace the envelope. Given the high densities in these regions we have assumed that the gas kinetic temperatures and the dust temperatures are equal.

The turbulent line widths were taken from Gaussian fits of the optically thin $\mathrm{C}^{17} \mathrm{O}$ data. The presence of hyperfine structure for the $\mathrm{C}^{17} \mathrm{O}$ was neglected for the models. By fixing the linewidth from our observations we were able to isolate the $\mathrm{CO}$ abundance relative to hydrogen as the free parameter. For simplicity we restricted our models to a simple uniform abundance of $\mathrm{C}^{17} \mathrm{O}$ throughout the envelope of each source. The input parameters for each model are given in Table 6.

These parameters were then used in conjunction with the adopted collisional rate coefficients of Flower (2001) (see also Schöier et al. 2005) to generate the predicted line emission. The $\mathrm{C}^{17} \mathrm{O}$ abundances were adjusted to produce the best match to the observed $\mathrm{C}^{17} \mathrm{O}$ line profiles. The modelled sources are circled in Fig. 3, illustrating that they cover a range dust and gas column densities and a range of implied $\mathrm{C}^{17} \mathrm{O}$ abundances.

The output spectra from RATRAN were continuum subtracted and convolved with a Gaussian with a FWHM equal to the appropriate JCMT beam for comparison with the observations. We analysed the quality of the models by using the reduced $\chi^{2}$ method, aligning the central channel of the model spectra with the peak of the Gaussian fit for the data. Figure 4 compares the observations with the results of the best fit models.

\subsection{Modelling results}

Figure 5 shows the success with which these (simple) models can match the observed $\mathrm{C}^{17} \mathrm{O}$ line profiles and also the sensitivity of the fit to the assumed $\mathrm{C}^{17} \mathrm{O}$ abundance. In this case the $\mathrm{C}^{17} \mathrm{O}$ abundance is constrained within the noise level of the observations to within $\sim 10 \%$.

Since RATRAN calculates the continuum emission in parallel with the line emission it was possible to check on the consistency of the models by comparing the predicted $850 \mu \mathrm{m}$ flux with that measured. This is especially important in this work where we are looking at the ratio of the line to continuum emission. For a number of sources modelled the predicted and measured $850 \mu \mathrm{m}$ flux differed by more than a factor of 3 . This is not completely surprising as the best fit models of WFS05 were selected to provide the best overall fit to the source SED and $850 \mu \mathrm{m}$ distribution, as such they do necessarily provide excellent fits to the $850 \mu \mathrm{m}$ flux alone. Therefore we have only considered the 8 sources where the predicted $850 \mu \mathrm{m}$ continuum emission differs from the measured value by less than a factor of 3 . The results for these sources are given in Table 7 .

\subsection{1. $\mathrm{C}^{17} \mathrm{O}$ analysis}

A comparison between the observed line profiles and the best fit model results for all the modelled sources is shown in Fig. 4. The models can successfully match the observed $\mathrm{C}^{17} \mathrm{O}$ line profile with a reasonable range of $\mathrm{C}^{17} \mathrm{O}$ fractional abundances ranging over the sample from $1.8 \times 10^{-8}$ to $1.5 \times 10^{-7}$. Importantly for the analysis of the sources for which models of the continuum emission do not exist, comparison of the $\mathrm{C}^{17} \mathrm{O}$ abundance inferred by the modelling and that derived directly from the observations agree within a factor of $\sim 2$ (Table 7). This result indicates that the assumptions made in directly estimating the $\mathrm{C}^{17} \mathrm{O}$ abundance from the observations are not overly biasing the results.

\subsection{2. $\mathrm{C}^{18} \mathrm{O}$ analysis}

For completeness we also attempted to model the $\mathrm{C}^{18} \mathrm{O}$ lines for the sources modelled in $\mathrm{C}^{17} \mathrm{O}$. Initial modelling of the $\mathrm{C}^{18} \mathrm{O}$ lines involved taking the best-fit $\mathrm{C}^{17} \mathrm{O}$ abundance and scaling it up by a range of potential values of the $\mathrm{C}^{18} \mathrm{O}$ to $\mathrm{C}^{17} \mathrm{O}$ abundance ratio; these were $R=2.8,3.65,4.0$ and 4.5 (as mentioned in Sect. 3.2). All other free parameters remain constant with the exception of the velocity dispersion which was adopted from the $\mathrm{C}^{17} \mathrm{O}$ hfs fit (as the best estimate of the intrinsic velocity dispersion within these regions). The results are also shown in Fig. 4. On inspection it is clear that a number of the models suffer from high optical depths, resulting in a flattening and broadening of the line shape, which is not seen in the observations. The inconsistencies between the data and the models for this isotopologue is perhaps not unexpected given the two velocity component profiles towards many of the sources which suggest that more than a simple envelope is contributing to the line profile.

An example of such a scenario would be a central core (as represented in our models), but surrounded by an extended low density envelope with the $\mathrm{C}^{18} \mathrm{O}$ having different optical depths in the envelope and in the core. The success of modelling the $\mathrm{C}^{17} \mathrm{O}$ from the core (Sect. 4.1) would suggest that the core has the higher optical depth.

For sources with two component $\mathrm{C}^{18} \mathrm{O}$ line profiles (Table 2), the $\mathrm{C}^{17} \mathrm{O}$ line is often intermediate in width between the two $\mathrm{C}^{18} \mathrm{O}$ components. This could also point towards the presence 


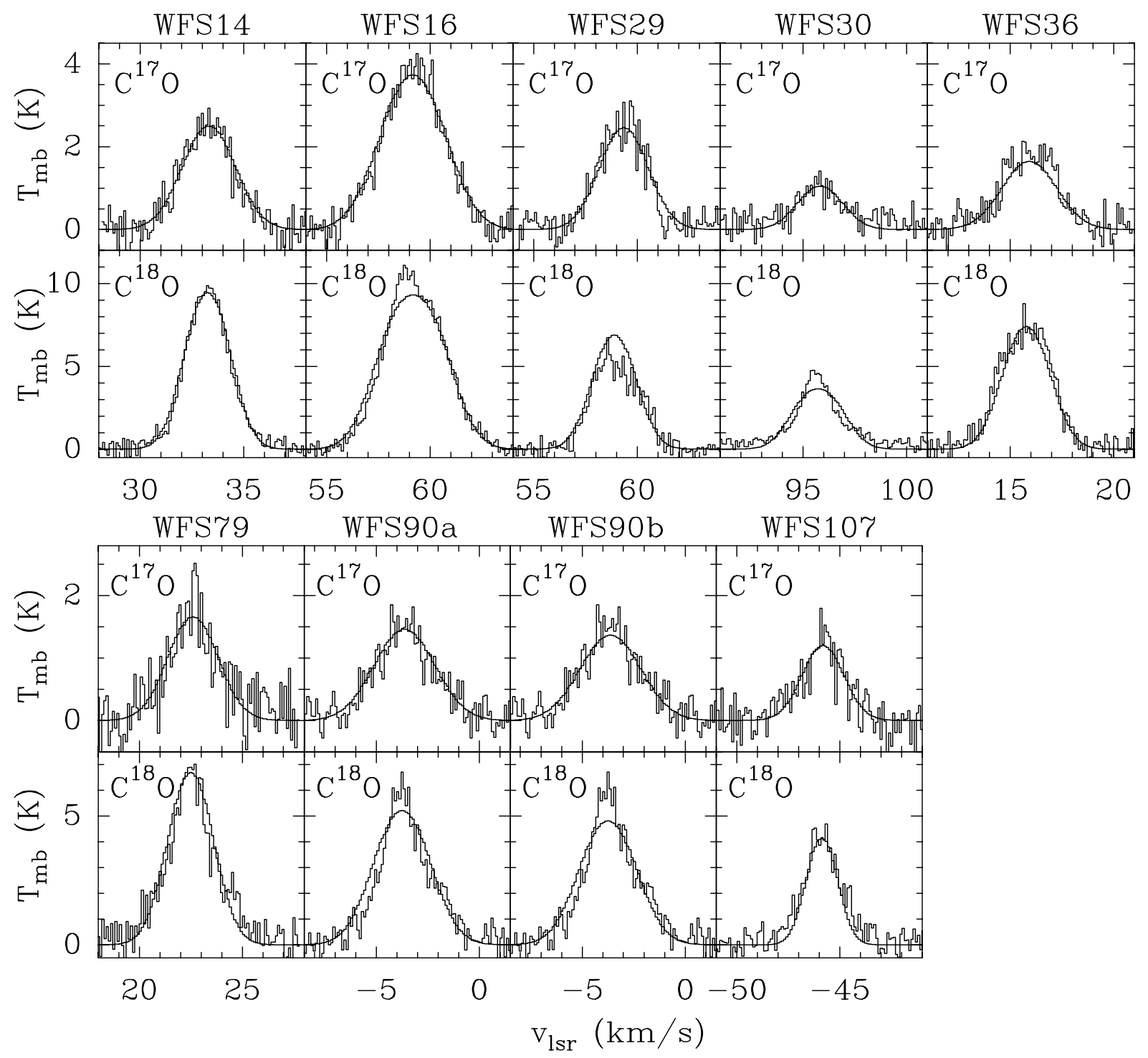

Fig. 4. Comparison of the $\mathrm{C}^{17} \mathrm{O} J=2 \rightarrow 1$ (upper spectra) and $\mathrm{C}^{18} \mathrm{O} J=2 \rightarrow 1$ (lower spectra) spectra with the best fit results of the RATRAN models (solid curves). The model parameters are given in Tables 6 and 7.

of two different components of material. If there are indeed two components then the simple column density analysis and model are actually overestimating the actual $\mathrm{C}^{17} \mathrm{O}$ and $\mathrm{C}^{18} \mathrm{O}$ column densities in the core, as some of the column density attributed to the core in a single component model is actually associated with the second component. This suggests that the abundances of these species in the core could be even lower than the values derived here indicate.

It is possible to test this prediction with maps of these sources; $\mathrm{C}^{18} \mathrm{O}$ emission extending beyond the detected $850 \mu \mathrm{m}$ emission would provide evidence for an outer envelope. It is possible that the relatively small chopper throw of the $850 \mu \mathrm{m}$ observations may have artificially removed this component from the SCUBA observations. This resulting underestimation of dust emission could account for the high abundances seen towards a few sources.

\section{Discussion}

The determination of the $\mathrm{C}^{17} \mathrm{O}$ column densities and hence abundances contain a number of sources of uncertainty. Since both the $\mathrm{C}^{17} \mathrm{O}$ lines are detected with good signal to noise ratios (Table 3, Fig. 4), and the dust continuum fluxes of the sources are well measured (WFS04), the statistical uncertainties due to noise are small. The calibration uncertainties may be larger, but are likely to affect all the sources equally. However the results do also depend on a number of other assumptions. Ideally all the sources observed should be modelled in detail to determine their $\mathrm{C}^{17} \mathrm{O}$ abundance throughout their envelopes and cores. However temperature and density profiles are not (yet) available for all the objects, so this is not currently practical. Nevertheless the objects which have been modelled in detail do suggest that systematic issues do not dominate the results. The similar $\mathrm{C}^{17} \mathrm{O}$ abundances derived from the modelling of a subsample of the sources and the $\mathrm{C}^{17} \mathrm{O}$ abundances derived directly from the observations, suggests that despite the approximations and assumptions it requires, the direct derivation produces reasonable estimates of the average $\mathrm{C}^{17} \mathrm{O}$ abundance towards the sources. It is these abundances on which we will focus.

To determine the origin of the scatter seen in Fig. 3 we must investigate the possible causes ranging from underlying physical differences to the simplifying assumptions. The consistency 


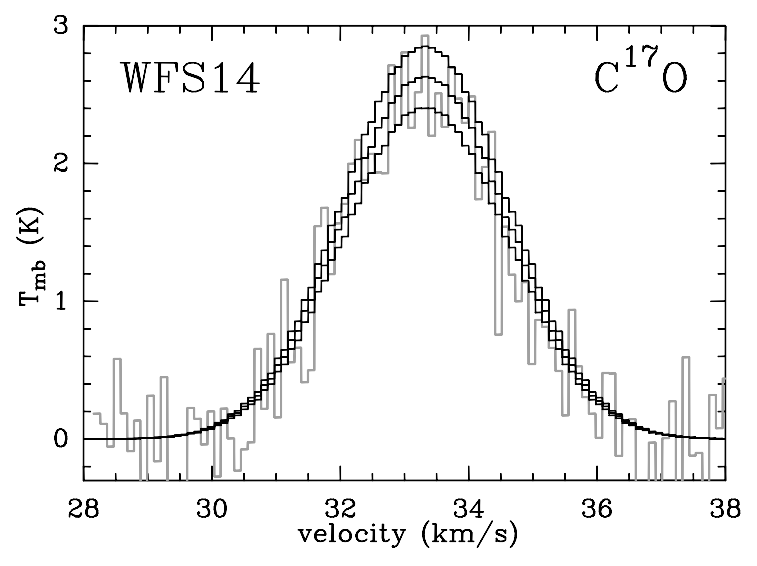

Fig. 5. The observed $\mathrm{C}^{17} \mathrm{O} J=2 \rightarrow 1$ emission towards WFS14 overlaid with the results of three models. The only difference between the three models is the assumed $\mathrm{C}^{17} \mathrm{O}$ abundances of $5 \times 10^{-8}, 5.5 \times 10^{-8}$ and $6 \times 10^{-8}$. The abundance is constrained within the noise level of the observations to within $\sim 10 \%$.

Table 7. Parameters of the best fit RATRAN models. Column 2 is the fractional abundance of $\mathrm{C}^{17} \mathrm{O}$ required for the model to match the observed $J=2 \rightarrow 1$ line profile. Column 3 is the reduced $\chi^{2}$ between the observed $\mathrm{C}^{17} \mathrm{O} J=2 \rightarrow 1$ transition and the modelled emission. $R\left(\mathrm{H}_{2}\right)$ is the ratio of measured $850 \mu \mathrm{m}$ flux to that generated by RATRAN. $R\left(\mathrm{C}^{17} \mathrm{O}\right)$ is the ratio of the best fit abundance from the modelling to the abundance derived from comparing the data to the $850 \mu \mathrm{m}$ hydrogen abundance: $\left[\mathrm{C}^{17} \mathrm{O} / \mathrm{H}_{2}\right]_{\bmod } /\left[\mathrm{C}^{17} \mathrm{O} / \mathrm{H}_{2}\right]_{\text {data }}$. Column 4 presents the best fit ratio of $\mathrm{C}^{18} \mathrm{O} / \mathrm{C}^{17} \mathrm{O}$ needed to match the $\mathrm{C}^{18} \mathrm{O}$ observations, scaling up the best-fit $\mathrm{C}^{17} \mathrm{O}$ abundances listed. The model line profiles are compared with the observations in Fig. 4.

\begin{tabular}{cccccc}
\hline \hline WFS & $\begin{array}{c}f\left(\mathrm{C}^{17} \mathrm{O}\right) \\
\left(10^{-8}\right)\end{array}$ & $\chi_{\text {red }}^{2}$ & {$\left[{ }^{18} \mathrm{O}\right] /\left[{ }^{17} \mathrm{O}\right]$} & $R\left(\mathrm{H}_{2}\right)$ & $R\left(\mathrm{C}^{17} \mathrm{O}\right)$ \\
\hline 14 & 5.2 & 1.69 & 4.0 & 1.36 & 1.35 \\
16 & 10.1 & 1.52 & 4.0 & 1.70 & 2.16 \\
29 & 10.0 & 1.79 & 2.8 & 0.76 & 1.00 \\
30 & 5.0 & 1.09 & 4.5 & 2.86 & 1.49 \\
36 & 15.0 & 1.58 & 4.5 & 1.30 & 1.88 \\
79 & 1.8 & 2.38 & 3.65 & 0.98 & 0.98 \\
$90 \mathrm{a}$ & 4.5 & 0.84 & 3.65 & 2.64 & 1.69 \\
$90 \mathrm{~b}$ & 4.5 & 0.86 & 3.65 & 2.15 & 1.69 \\
107 & 4.0 & 1.13 & 2.8 & 1.04 & 0.94 \\
\hline
\end{tabular}

within a factor of 2 of the $\mathrm{C}^{17} \mathrm{O}$ abundances generated using RATRAN and those derived straight from the data suggests that the scatter between the observed $\mathrm{C}^{17} \mathrm{O}$ column density and $\mathrm{H}_{2}$ column density is not a result of an overly simplistic data analysis or the assumed excitation temperature.

It is important to recognise that the hydrogen column density derived from the dust emission suffers from uncertainties due to the assumed dust properties. In calculating the dust column density we have adopted a constant value of $\kappa$, the dust mass opacity, for all the sources. For our calculations we adopted a value from Ossenkopf \& Henning (1994) assuming a gas density of $10^{6} \mathrm{~cm}^{-3}$, with thin ice mantles and $10^{5}$ years of coagulation. However we investigated the influence of this assumption by recalculating $N\left(\mathrm{H}_{2}\right)$ for the full range of possible opacities from Ossenkopf \& Henning. By manipulating the opacity it is possible to move the entire sample in Fig. 3 to a range either above the canonical $\mathrm{C}^{17} \mathrm{O}$ abundance of $4.7 \times 10^{-8}$ or below it. However assuming that all the sources should lie at this value, it is clear that changing the dust in order to allow that to happen would have to be done on a source by source basis and utilising the full range of potential opacities.

For those sources that lie to the left of the canonical abundance line, an opacity corresponding to grains that have not undergone any coagulation would need to be adopted. This lack of grain coagulation would seem to imply that these sources, and the dust surrounding them, are young, not yet having had time for the dust to coagulate. To the other side of the line lie the sources which appear to be the most depleted of our sample. To change their abundances would require an opacity corresponding to some degree of coagulation and for the most under-abundant sources this would need to include an absence of ice mantles. The scenario of coagulated grains without ice would be consistent with the dust close to a star which has heated its surroundings and evaporated all icy mantles. However the temperature profiles derived by DUSTY modelling show that only a small part of the envelopes are this warm, with the bulk of each envelope lying at temperatures below $50 \mathrm{~K}$. Under these conditions we would consider the complete absence of ice mantles to be an unreasonable assumption. The dust models indicate if mantles of any kind are present, they would limit the change in the inferred hydrogen column density to not more than $12 \%$ of the calculated values.

In addition, van der Tak et al. (1999) found that by comparing the dust mass from FIR and submm data to the gas mass derived from regions without depletion of $\mathrm{C}^{17} \mathrm{O}$, only the opacities for dust grains with ice mantles reproduced the standard dust-to-gas ratio of 1:100. Additionally Fig. 3 shows a number of the sources having abundances in excess of $4.7 \times 10^{-8}$, which if not due to particular circumstances for these objects, could indicate that for the sample as a whole the hydrogen column density could be underestimated. Given these points we conclude that the scatter seen between the abundances does indeed reflect physical differences between the sources.

In principle, selective photodestruction of the $\mathrm{C}^{17} \mathrm{O}$ in the inner regions of the sources close to the central heating sources could give rise to the varying $\mathrm{C}^{17} \mathrm{O}$ abundance. However selective photodestruction can only be an issue in the region up to optical depth $\sim 1$ in the UV from the inner edge of the circumstellar envelope. Although this optical depth can be contributed by either dust or line self-shielding, since the circumstellar envelopes are dusty, it is the dust that will be the dominant UV opacity source. This UV opacity corresponds to a visual optical depth $\sim 0.1$ in the optical. Since this corresponds to about $1 \%$ of the thickness of the envelope (WFS04), the internal UV flux cannot be affecting the $\mathrm{C}^{17} \mathrm{O}$ abundance by more than $\sim 1 \%$.

The low optical depth of the $\mathrm{C}^{17} \mathrm{O}$ transitions suggest that if $\mathrm{C}^{17} \mathrm{O}$ is uniformly abundant throughout each source, the $\mathrm{C}^{17} \mathrm{O}$ emission, and hence $\mathrm{C}^{17} \mathrm{O}$ column density should, like the dust emission, trace the total column density even though the $\mathrm{C}^{17} \mathrm{O}$ has a relatively low critical density.

The most likely explanation for the variation in the $\mathrm{C}^{17} \mathrm{O}$ abundance appears to be that it reflects different gas phase abundance of $\mathrm{C}^{17} \mathrm{O}$ due to its freeze out onto dust grains. Such depletion of $\mathrm{C}^{17} \mathrm{O}$ (and by implication, $\mathrm{CO}$ and possibly other species) towards similar high mass sources has been previously reported by VVEB and Fontani et al. (2006).

\subsection{Depletion of $\mathrm{C}^{17} \mathrm{O}$}

If the scatter in the $\mathrm{C}^{17} \mathrm{O}$ abundance is indeed due to depletion, then one might expect that the regions with the coolest dust would have the highest depletion. VVEB investigated this using 
Table 8. Mass-weighted temperatures in Kelvin, calculated for those sources for which the data are available.

\begin{tabular}{cccccccc}
\hline \hline WFS & $\bar{T}_{\mathrm{mw}}$ & WFS & $\bar{T}_{\mathrm{mw}}$ & WFS & $\bar{T}_{\mathrm{mw}}$ & WFS & $\bar{T}_{\mathrm{mw}}$ \\
\hline 6 & 23.2 & 19 & 23.5 & 66 & 26.5 & 95 & 29.7 \\
13 & 25.4 & 25 & 18.6 & 78 & 18.6 & 107 & 35.3 \\
14 & 28.5 & 29 & 34.1 & 79 & 28.4 & 108 & 20.8 \\
16 & 23.4 & 30 & 23.4 & 80 & 27.9 & 109 & 18.4 \\
18 & 18.6 & 36 & 30.0 & 90 & 25.4 & 110 & 20.8 \\
\hline
\end{tabular}

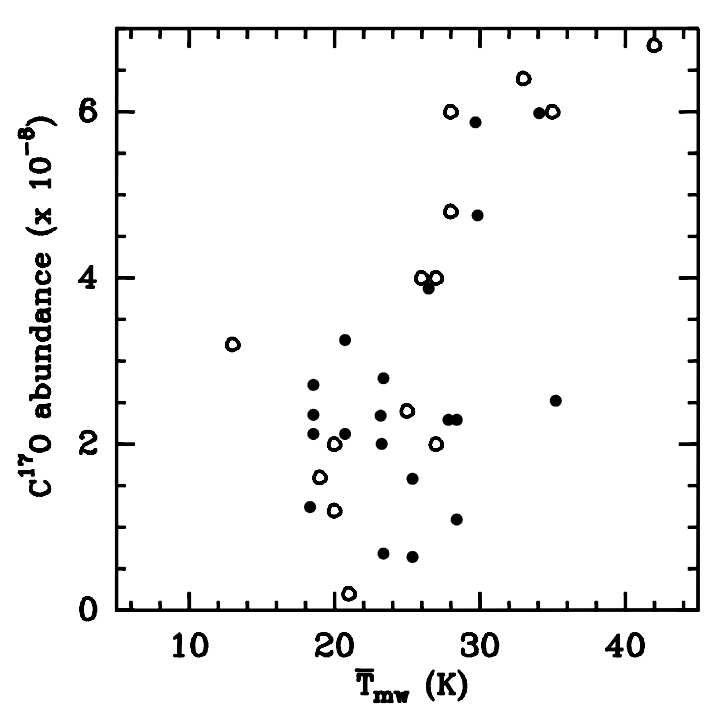

Fig. 6. $\mathrm{C}^{17} \mathrm{O} J=2 \rightarrow 1$ abundance against the mass-weighted temperature, $\bar{T}_{\mathrm{mw}}$. The solid dots denote our data whilst the circles represent the data from Fig. 11 in VVEB.

the mass-weighted temperature, $\bar{T}_{\mathrm{mw}}$, to define a characteristic temperature for each source.

Figure 6 shows the $\mathrm{C}^{17} \mathrm{O}$ abundance against the massweighted temperature for all the sources for which the necessary parameters were available (Table 8). The mass-weighted temperature is defined by VVEB and was calculated here using the temperature profile from the DUSTY modelling of the sources. The figure shows data points from both our sources and those from VVEB. The two data sets are in reasonable agreement, with the distribution of both being consistent with depletion at low temperatures. The Spearman correlation coefficient indicates that the $\mathrm{C}^{17} \mathrm{O}$ abundance and mass-weighted temperature are correlated at a $96 \%$ confidence level.

We do however see a number of our sources exhibiting higher abundances at lower temperatures than VVEB, but none with showing the reverse. This is consistent with an absence of depletion at higher temperatures, whilst the sources at low temperature and high abundance could be explained by having additional contributions of $\mathrm{C}^{17} \mathrm{O}$ for regions outside the modelled core. This would be consistent with the $\mathrm{C}^{17} \mathrm{O}$ linewidths lying intermediate between the two components of the $\mathrm{C}^{18} \mathrm{O}$ lines as discussed above.

The degree of depletion in a region can provide an estimate of the time for which gas has been cold and at high density. This provides a measure of timescales for the formation of high-mass stars. Models of the physical and chemical changes occurring in the gas and on dust grains during the pre-protostellar phase of the evolution of cores, lead to predictions for the expected abundances of molecules such as CO as a function of time (Viti \& Williams 1999; Flower et al. 2005). With detailed knowledge

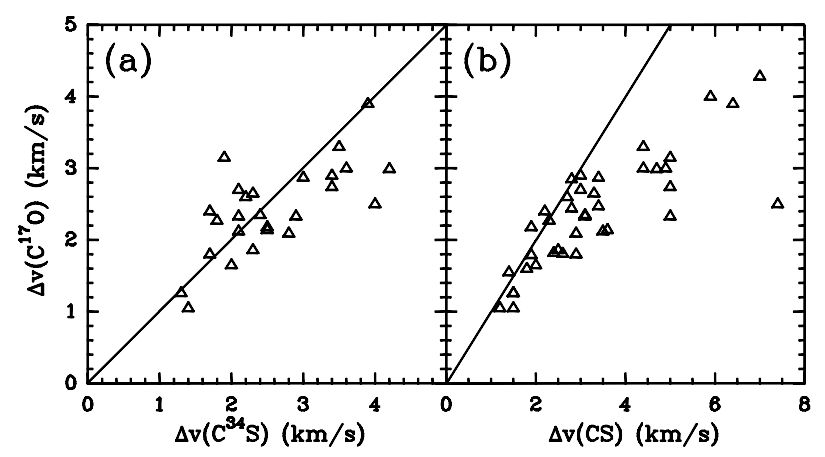

Fig. 7. Plot showing the $\mathrm{C}^{17} \mathrm{O}$ hfs linewidth against a) the $\mathrm{C}^{34} \mathrm{~S}$ $J=3 \rightarrow 2$ and b) the CS $J=3 \rightarrow 2$ linewidths (Beuther et al. 2002a). The solid lines show the unity ratio.

of depletion levels they become a viable tool for determining the ages of the cores.

The detection of both $\mathrm{C}^{17} \mathrm{O}$ and $\mathrm{C}^{18} \mathrm{O}$ towards all of the sample shows that that there are no sources towards which essentially all $\mathrm{CO}$ has been depleted. This places an upper limit on the time for which the dust can have existed at low temperatures of $T<10 \mathrm{~K}$. As we do not find depletion to exceed more than a factor of $\sim 10$ we can use the abundances predicted by Flower et al. (2005) to estimate that the cores being traced are not older than $\sim 3.5 \times 10^{5}$ years. (For a depletion factor of 5 this reduces to $\sim 2.2 \times 10^{5}$ years.)

\subsection{Evidence of source evolution?}

This set of sources has also been observed by Beuther et al. (2002a) in $1.2 \mathrm{~mm}$ continuum emission and a number of CS lines. Figure 7 shows a comparison of the linewidth of $\mathrm{C}^{17} \mathrm{O} J=2 \rightarrow 1$ (determined from the fit to the hyperfine structure of the transition) against the linewidths of CS $J=3 \rightarrow 2$ and $\mathrm{C}^{34} \mathrm{~S} J=3 \rightarrow 2$. It is clear that at smaller linewidths the two are most closely correlated, with approximately equal linewidths. However, the linewidths tend to have a larger ratio at larger linewidths. For CS linewidths $<3 \mathrm{~km} \mathrm{~s}^{-1}$ the ratio of the $\mathrm{CS}$ linewidth to the $\mathrm{C}^{17} \mathrm{O}$ linewidth is $1.17 \pm 0.05$, while for CS linewidths $\geq 3 \mathrm{~km} \mathrm{~s}^{-1}$, the ratio is $1.56 \pm 0.09$. The same trend is also seen in the rarer species $\mathrm{C}^{34} \mathrm{~S}$, where the ratio for $\mathrm{C}^{34} \mathrm{~S}$ linewidths $<3 \mathrm{~km} \mathrm{~s}^{-1}$ is $1.00 \pm 0.05$ and $1.22 \pm 0.07$ for the larger linewidth sources, suggesting this is not a result of high optical depth in the CS line. We speculate that this increasing ratio of $\mathrm{CS}$ to $\mathrm{C}^{17} \mathrm{O}$ linewidth could arise as a result of $\mathrm{CS}$ being more affected than the $\mathrm{C}^{17} \mathrm{O}$ by heating and stirring of the material by the central source, through the action of the outflow.

Interestingly there appears to be a connection between the $\mathrm{C}^{17} \mathrm{O}$ abundance towards a source and its $\mathrm{CS}$ linewidth. Comparing the distributions of $\mathrm{C}^{17} \mathrm{O}$ abundance for those sources with $\Delta v(\mathrm{CS})>3 \mathrm{~km} \mathrm{~s}^{-1}$ and those for which $\Delta v(\mathrm{CS})<$ $3 \mathrm{~km} \mathrm{~s}^{-1}$, a Kolmogorov-Smirnov two-sample test indicates a $98.6 \%$ likelihood of a difference between the two groups (see Fig. 8a). We also considered just the upper $(\Delta v(\mathrm{CS}) \gg$ $\left.3 \mathrm{~km} \mathrm{~s}^{-1}\right) \sim 30 \%$ of the sample, comparing its distribution of $\mathrm{C}^{17} \mathrm{O}$ abundance with that of the lower $(\Delta v(\mathrm{CS}) \ll$ $\left.3 \mathrm{~km} \mathrm{~s}^{-1}\right) \sim 30 \%$ of the sample. Repeating the K-S test for these groups of sources increases the statistical significant of the difference between these new groups to $99.7 \%$ (see Fig. 8b), despite the smaller number of sources in each group. This increase in the difference between the groups might be expected if these groups are not distinct categories but rather represent two extremes in a 


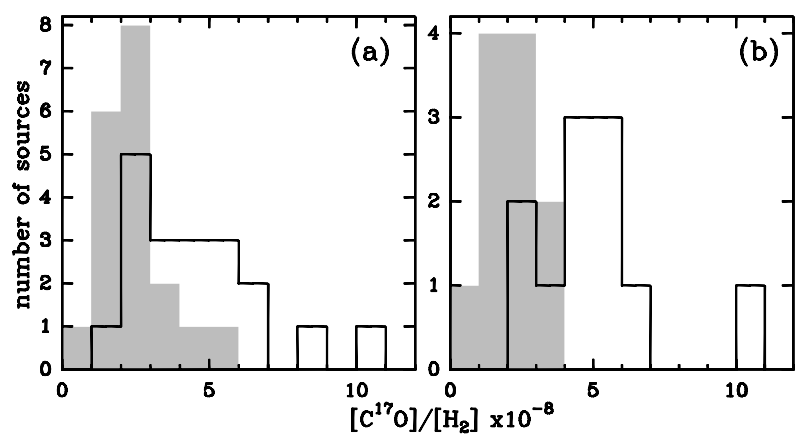

Fig. 8. Distribution of $\mathrm{C}^{17} \mathrm{O}$ abundance. a) The solid bars show the distribution of abundances for those sources with $\Delta v(\mathrm{CS})>3 \mathrm{~km} \mathrm{~s}^{-1}$ whilst the hollow bars represent those sources with $\Delta v(\mathrm{CS})<3 \mathrm{~km} \mathrm{~s}^{-1}$. b) The solid bars show the distribution of abundances for the top 30\% of the sample $\left(\Delta v(\mathrm{CS}) \gg 3 \mathrm{~km} \mathrm{~s}^{-1}\right)$ while the hollow bars show the lower $30 \%$ of the sample $\left(\Delta v(\mathrm{CS}) \ll 3 \mathrm{~km} \mathrm{~s}^{-1}\right)$.

continuum of source properties, from small linewidth, high $\mathrm{C}^{17} \mathrm{O}$ abundance objects to large linewidth, low $\mathrm{C}^{17} \mathrm{O}$ abundance objects, perhaps tracing an evolutionary progression.

To further probe the statistical significance of this difference between the sources we performed two tests. In the first we randomly shuffled the $\mathrm{C}^{17} \mathrm{O}$ abundances amongst the sources. The sample was then again divided between objects with $\Delta v(\mathrm{CS})>$ $3 \mathrm{~km} \mathrm{~s}^{-1}$ and those for which $\Delta v(\mathrm{CS})<3 \mathrm{~km} \mathrm{~s}^{-1}$. A K-S test was then performed comparing the resulting distributions of $\mathrm{C}^{17} \mathrm{O}$ abundance for the two linewidth groups. Repeating this randomisation and testing 100 times we found no cases where the statistical difference between the shuffled abundances exceed that of actual data. In other words the difference in $\mathrm{C}^{17} \mathrm{O}$ abundance between the sources with large linewidths and those with small linewidths has a probability of less than $1 \%$ of being a chance coincidence.

For the second test, we take into account the observational uncertainties associated with the abundances by randomly resampling the abundances associated with each source. For each source, we regenerated a $\mathrm{C}^{17} \mathrm{O}$ abundance by randomly drawing an abundance from a Gaussian distribution with a dispersion equal to the uncertainty in the measured $\mathrm{C}^{17} \mathrm{O}$ abundance of that source. We did this for the whole sample, split the sample on the basis of the CS linewidth and again apply a K-S test to intercompare the resulting distributions of $\mathrm{C}^{17} \mathrm{O}$ abundance. This was repeated 100 times. We found that on average the two samples differed at $98 \%$ confidence level, with the confidence level never less than $97 \%$. This indicates that the difference in abundance between our two groups is unlikely to arise as a result of the statistical uncertainties.

The association of low $\mathrm{C}^{17} \mathrm{O}$ abundance with sources with large linewidths is somewhat surprising. However it can be understood if the low $\mathrm{C}^{17} \mathrm{O}$ abundance (which is calculated integrated across the whole line profile and averaged across the telescope beam) reflects the conditions in the cold environment in the outer, extended envelope around the central source, while the broad linewidth reflects some component close to the central source, possibly related to outflow. Mapping the spatial distribution of the depletion towards these objects by comparing molecular line and deep dust continuum maps would be able to test this interpretation.

Whilst the central regions of these sources certainly have temperatures reflecting the presence of a luminous embedded source, the models of WFS05 imply that the majority of the extended envelope lies at temperatures below $50 \mathrm{~K}$. In many cases the models show the temperature dropping below $20 \mathrm{~K}$ towards the outer regions of the envelopes, temperatures which are similar to those derived from the $\mathrm{NH}_{3}$ observations of SBSMW. It is presumably in these outer regions, far from the heating and effects of the outflow from the central source, that the $\mathrm{C}^{17} \mathrm{O}$ is frozen out onto the surface of dust grains.

The high $\mathrm{C}^{17} \mathrm{O}$ abundances displayed by those sources with the lowest linewidths could be consistent with two possible scenarios; either these sources are extremely young and depletion has not yet occurred to a significant degree, or alternatively the sources are much older having undergone substantial heating by an embedded population of sources, or shocks from outflows, which has evaporated the ice mantles containing the $\mathrm{C}^{17} \mathrm{O}$ back into the gas phase.

This second scenario could be confirmed by the positive detection towards these sources of the molecules which trace hot core emission. SBSMW have searched many of these sources for such species and shown that at least some of these sources are evolved enough to have locally heated their natal cores to temperatures of $>100 \mathrm{~K}$. However the failure to detect these molecules towards some sources implies that these sources are either highly evolved with the hot core molecules have been destroyed by ion reactions and ages $\sim 10^{5}$ years (e.g. Hatchell et al. 1998), or else would suggest that these are extremely young sources. A more detailed discussion of these possible scenarios is presented in Thomas \& Fuller (2007). High spatial resolution observations of these regions would help to distinguish between the two pictures by identifying the location of the depleted material. Very young cores would be expected to be depleted in $\mathrm{C}^{17} \mathrm{O}$ in their centres where the density is highest but to have normal $\mathrm{C}^{17} \mathrm{O}$ abundance in their more extended, lower density, envelopes. For a more evolved core which has formed a high mass protostar at its centre, this central object will be heating the interior region of the core, releasing frozen out $\mathrm{C}^{17} \mathrm{O}$ back into the gas phase. However sufficient time may have passed for $\mathrm{C}^{17} \mathrm{O}$ to be depleted in the outer parts of the core.

\section{Summary}

We have presented spectra of 84 candidate HMPOs in the $J=2 \rightarrow 1$ line of $\mathrm{C}^{17} \mathrm{O}$ and for a selection of these we have also observed $\mathrm{C}^{18} \mathrm{O} J=2 \rightarrow 1$ and $\mathrm{C}^{17} \mathrm{O} J=3 \rightarrow 2$. We have used the data to calculate the $\mathrm{C}^{17} \mathrm{O}$ column densities and for those sources for which models of the dust emission exist, we constructed models of the $\mathrm{CO}$ emission to check the validity of our assumptions regarding excitation temperature and dust temperature. We have found the following:

The ratio of $\mathrm{C}^{17} \mathrm{O}$ column density to $\mathrm{H}_{2}$ derived from $850 \mu \mathrm{m}$ dust emission has a significant scatter across our sample. The scatter between the most extreme sources is $\sim 14$ and is consistent with the abundance of $\mathrm{C}^{17} \mathrm{O}$ differing on a source-by-source basis.

Our derivation of the $\mathrm{H}_{2}$ column density assumed a constant value for the dust opacity $\kappa$, for all the sources, however there are no corrections to $\kappa$ which when applied globally to the sample eliminate this scatter. Indeed the elimination of the scatter would require the sources lying at the extremes of the scatter to possess dust properties which are physically unlikely.

The method and assumptions used to calculate the $\mathrm{C}^{17} \mathrm{O}$ column densities were tested by modelling a subset of these sources with the radiative transfer code RATRAN. For all suitable models the best-fit abundance of $\mathrm{C}^{17} \mathrm{O}$ matched the data to within a factor of $\sim 2$, supporting our assumptions and removing the possibility of the assumptions accounting for the range of 
abundances. We conclude that the variation in abundance arises as a result of depletion present in a number of these sources. However the maximum degree of depletion we find does not exceed $\sim 7$. This range of depletion suggests that outer regions of these sources have lifetimes during which they are cold and dense of about $2-4 \times 10^{5}$ years.

We derive the mass-weighted temperature and find a correlation with $\mathrm{C}^{17} \mathrm{O}$ abundance, consistent with VVEB who from this correlation also infer the presence of depletion towards sources similar to those discussed here.

These sources have been studied by Beuther et al. (2002a) and a comparison of our $\mathrm{C}^{17} \mathrm{O}$ linewidths to their $\mathrm{CS} J=3 \rightarrow 2$ linewidths shows a division between those which have $\Delta v(\mathrm{CS})>3 \mathrm{~km} \mathrm{~s}^{-1}$ and those for which $\Delta v(\mathrm{CS})<3 \mathrm{~km} \mathrm{~s}^{-1}$. A statistical comparison of the $\mathrm{C}^{17} \mathrm{O}$ abundances of these two groups reveal a trend with the first group displaying significantly lower abundances than those in the second group with a statistical significance exceeding $\sim 98 \%$.

We suggest that this range of $\mathrm{C}^{17} \mathrm{O}$ abundance and the implied degree of depletion between the sources may reflect a spread in evolutionary status amongst the sources. Higher angular resolution observations of dust emission, $\mathrm{C}^{17} \mathrm{O}$ and other species towards these objects will be important in confirming this suggestion. It is possible that high resolution observations will identify regions with higher degrees of depletion than can be measured with the relatively large telescope beam used for the observations presented here.

Depletion towards these objects shows that during the evolution of these cores the gas has remained cold and dense for long enough for the trace species to deplete. More detailed models of how trace molecules deplete in massive dense cores, combined with higher angular resolution observations, should be able to provide tighter constraints on the lifetime of these regions, including how long they exist before they form massive stars.

\section{References}

Bensch, F., Pak, I., Wouterloot, J. G. A., Klapper, G., \& Winnewisser, G. 2001, ApJ, 562, 185

Beuther, H., Schilke, P., Menten, K. M., et al. 2002a, ApJ, 566, 945
Beuther, H., Schilke, P., Sridharan, T. K., et al. 2002b, A\&A, 383, 892

Caselli, P., Walmsley, C. M., Tafalla, M., Dore, L., \& Myers, P. C. 1999, ApJ, 523, L165

Cesaroni, R., Galli, D., Lodato, G., Walmsley, C. M., \& Zhang, Q. 2007, Protostars and Planets V, ed. B. Reipurth, D. Jewitt, \& K. Keil (Tucson: University of Arizona Press), 197

Flower, D. R. 2001, JPhB, 34, 2731

Flower, D. R., Pineau Des Forêts, G., \& Walmsley, C. M. 2005, A\&A, 436, 933

Fontani, F., Caselli, P., Crapsi, A., et al. 2006, A\&A, 460, 709

Frerking, M. A., Langer, W. D., \& Wilson, R. W. 1982, ApJ, 262, 590

Fuller, G. A., Williams, S. J., \& Sridharan, T. K. 2005, A\&A, 442, 949

Hatchell, J., Thompson, M. A., Millar, T. J., \& MacDonald, G. H. 1998, A\&A, 338,713

Hildebrand, R. H. 1983, QJRAS, 24, 267

Hogerheijde, M. R., \& van der Tak, F. F. S. 2000, A\&A, 362, 697

Ivezić, Ž., Nenkova, M., \& Elitzur, M. 1999, User Manual for DUSTY, University of Kentucky Internal Report

Kramer, C., Alves, J., Lada, C. J., et al. 1999, A\&A, 342, 257

Kurtz, S., Cesaroni, R., Churchwell, E., Hofner, P., \& Walmsley, C. M. 2000, Protostars \& Planets IV (Tucson: Univ. Arizona Press), 299

Ladd, E. F. 2004, ApJ, 610, 320

Ladd, E. F., Fuller, G. A., \& Deane, J. R. 1998, ApJ, 495, 871

Ossenkopf, V., \& Henning, Th. 1994, A\&A, 291, 943

Penzias, A. A. 1981, ApJ, 249, 518

Redman, M. P., Rawlings, J. M. C., Nutter, D. J., Ward-Thompson, D., \& Williams, D. A. 2002, MNRAS, 337, L17

Savva, D., Little, L. T., Phillips, R. R., \& Gibb, A. G. 2003, MNRAS, 343, 259

Schöier, F. L, van der Tak, F. F. S., van Dishoeck, E. F., \& Black, J. H. 2005, A\&A, 432, 369

Sheffer, Y., Lambert, D. L., \& Federman, S. R. 2002, ApJ, 574, 171

Shepherd, D. 2005, Massive Star Birth: A Crossroads of Astrophysics, ed. R. Cesaroni, M. Felli, E. Churchwell, \& M. Walmsley (Cambridge: Cambridge University Press), IAU Symp., 227, 237

Sridharan, T. K., Beuther, H., Schilke, P., Menten, K. M., \& Wyrowski, F. 2002, ApJ, 566, 933 (SBSMW)

Tafalla, M., Myers, P. C., Caselli, P., Walmsley, C. M., \& Comito, C. 2002, ApJ, 569,815

Thomas, H. S., \& Fuller, G. A. 2007, ApJ, 659, L165

van der Tak, F. F. S., van Dishoeck, E. F., Evans, N. J., Bakker, E. J., \& Blake, G. A. 1999, ApJ, 522, 911

van der Tak, F. F. S., van Dishoeck, E. F., Evans, N. J. II, \& Blake, G. A. 2000, ApJ, 537, 283 (VVEB)

Viti, S., \& Williams, D. A. 1999, MNRAS, 305, 755

Willacy, K., Langer, W. D., \& Velusamy, T. 1998, ApJ, 507, L171

Williams, S. J., Fuller, G. A., \& Sridharan, T. K. 2004, A\&A, 417, 115 (WFS04)

Williams, S. J., Fuller, G. A., \& Sridharan, T. K. 2005, A\&A, 434, 257 (WFS05)

Wouterloot, J. G. A., Brand, J., \& Henkel, C. 2005, A\&A, 430, 549 
H. S. Thomas and G. A. Fuller: HMPOs: depletion, Online Material $p 1$

Table 1. The telescope pointing coordinates are given along with the name of the associated IRAS source. The WFS labels refer to the submm peaks identified in Williams et al. (2004).

\begin{tabular}{|c|c|c|c|c|c|c|}
\hline \multirow[t]{2}{*}{ WFS } & \multirow[t]{2}{*}{ IRAS name } & \multirow{2}{*}{$\frac{\text { RA }}{(\mathrm{J} 2000)}$} & \multirow{2}{*}{$\begin{array}{c}\text { Dec } \\
(\mathrm{J} 2000)\end{array}$} & \multicolumn{3}{|c|}{ Dates of observations } \\
\hline & & & & $\mathrm{C}^{18} \mathrm{O}(2-1)$ & $\mathrm{C}^{17} \mathrm{O}(2-1)$ & $\mathrm{C}^{17} \mathrm{O}(3-2)$ \\
\hline WFS1 & IRAS $05358+3543$ & 053910.8 & +354516 & & 05/07/21 & $05 / 07 / 23$ \\
\hline WFS3 & IRAS $05490+2658$ & 055211.0 & +270034 & & $05 / 07 / 21$ & $05 / 07 / 23$ \\
\hline WFS4 & IRAS $05490+2658$ & 055212.1 & +270011 & & $05 / 07 / 21$ & $05 / 07 / 23$ \\
\hline WFS6 & IRAS $05553+1631$ & 055813.4 & +163200 & & $05 / 07 / 21$ & $05 / 07 / 23$ \\
\hline WFS7 & IRAS 18089-1732 & 181145.2 & -173043 & & & 05/07/07 \\
\hline WFS8 & IRAS 18089-1732 & 181151.5 & -173134 & & & $05 / 07 / 07$ \\
\hline WFS11 & IRAS 18089-1732 & 181157.0 & -172934 & & & 05/07/07 \\
\hline WFS12 & IRAS 18090-1832 & 181202.1 & -183158 & & $05 / 08 / 03$ & \\
\hline WFS13 & IRAS 18102-1800 & 181311.7 & -180004 & & 05/08/03 & \\
\hline WFS14 & IRAS 18151-1208 & 181758.2 & -120728 & $04 / 05 / 13$ & $04 / 05 / 13,04 / 05 / 14$ & \\
\hline WFS15 & IRAS 18159-1550 & 181848.4 & -154900 & & $05 / 08 / 03$ & \\
\hline WFS16 & IRAS 18182-1433 & 182108.9 & -143146 & $04 / 05 / 13$ & $04 / 05 / 13$ & \\
\hline WFS17 & IRAS 18223-1243 & 182510.6 & -124227 & $04 / 05 / 13$ & $04 / 05 / 14$ & \\
\hline WFS18 & IRAS 18247-1147 & 182731.4 & -114555 & & $05 / 07 / 27,05 / 08 / 03$ & \\
\hline WFS19 & IRAS 18264-1152 & 182914.3 & -115022 & & $05 / 07 / 27,05 / 08 / 03$ & \\
\hline WFS20 & IRAS 18272-1217 & 183002.2 & -121540 & $04 / 05 / 13$ & $04 / 05 / 14$ & \\
\hline WFS 21 & IRAS 18272-1217 & 183003.2 & -121511 & $04 / 05 / 13$ & $04 / 05 / 14$ & \\
\hline WFS22 & IRAS 18290-0924 & 183143.4 & -092226 & $04 / 05 / 14$ & $04 / 05 / 14$ & $05 / 07 / 22$ \\
\hline WFS23 & IRAS 18290-0924 & 183144.0 & -092217 & & $05 / 08 / 03$ & \\
\hline WFS24 & IRAS 18306-0835 & 183317.3 & -083328 & & 05/08/03 & \\
\hline WFS 25 & IRAS 18306-0835 & 183323.9 & -083333 & $04 / 05 / 13$ & $04 / 05 / 13$ & \\
\hline WFS28 & IRAS 18310-0825 & 183347.9 & -082352 & & $05 / 08 / 03$ & \\
\hline WFS29 & IRAS 18337-0743 & 183627.9 & -074025 & $04 / 05 / 14$ & $04 / 05 / 14$ & $05 / 07 / 22$ \\
\hline WFS30 & IRAS 18345-0641 & 183716.8 & -063835 & $04 / 05 / 13$ & $04 / 05 / 13$ & \\
\hline WFS33 & IRAS 18348-0616 & 183730.5 & -061413 & & 05/07/27, 05/08/03 & \\
\hline WFS34 & IRAS 18372-0541 & 183716.8 & -063835 & $04 / 05 / 14,04 / 05 / 15$ & $04 / 05 / 14$ & \\
\hline WFS35 & IRAS 18385-0512 & 184112.8 & -050858 & & 05/08/03 & \\
\hline WFS36 & IRAS 18426-0204 & 184512.1 & -020110 & $04 / 05 / 14,04 / 05 / 15$ & $04 / 05 / 14$ & \\
\hline WFS37 & IRAS 18431-0312 & 184545.5 & -030921 & & $05 / 07 / 27,05 / 08 / 03$ & \\
\hline WFS38 & IRAS 18437-0216 & 184621.8 & -021220 & & $05 / 07 / 27,05 / 08 / 03$ & \\
\hline WFS39 & IRAS 18437-0216 & 184622.4 & -021416 & $04 / 05 / 14,04 / 05 / 15$ & $04 / 05 / 14,04 / 05 / 15$ & 05/07/07 \\
\hline WFS41 & IRAS 18440-0148 & 184633.3 & -014452 & & $05 / 08 / 03$ & \\
\hline WFS42 & IRAS 18440-0148 & 184636.5 & -014522 & & $05 / 08 / 03$ & \\
\hline WFS51 & IRAS 18460-0307 & 184837.8 & -030348 & $04 / 05 / 14,04 / 05 / 15$ & $04 / 05 / 14,04 / 05 / 15$ & 05/07/07 \\
\hline WFS55 & IRAS 18472-0022 & 184952.4 & -001859 & & $05 / 08 / 03$ & \\
\hline WFS57 & IRAS $18488+0000$ & 185124.4 & +000439 & & $05 / 07 / 26$ & \\
\hline WFS58 & IRAS $18488+0000$ & 185125.5 & +000411 & & $05 / 07 / 26$ & \\
\hline WFS59 & IRAS $18521+0134$ & 185440.6 & +013805 & & 05/08/03 & \\
\hline WFS60 & IRAS $18521+0134$ & 185444.4 & +013700 & & 05/08/03 & \\
\hline WFS61 & IRAS $18530+0215$ & 185533.7 & +021909 & & $05 / 08 / 03$ & \\
\hline WFS62 & IRAS $18540+0220$ & 185636.6 & +022445 & & $05 / 07 / 30$ & \\
\hline WFS63 & IRAS $18540+0220$ & 185640.1 & +022530 & & $05 / 07 / 30$ & \\
\hline WFS64 & IRAS $18553+0414$ & 185753.5 & +041816 & & $05 / 07 / 30$ & \\
\hline WFS66 & IRAS $19012+0536$ & 190345.3 & +054043 & & 05/08/03 & \\
\hline WFS67 & IRAS 19035+0641 & 190601.5 & +064635 & & $05 / 08 / 03$ & \\
\hline WFS68 & IRAS $19074+0752$ & 190953.4 & +075712 & & $05 / 07 / 29$ & \\
\hline WFS69 & IRAS $19074+0752$ & 190953.9 & +075655 & & $05 / 07 / 29$ & \\
\hline WFS70 & IRAS $19175+1357$ & 191948.6 & +140226 & & $05 / 07 / 27$ & \\
\hline WFS71 & IRAS $19175+1357$ & 191948.8 & +140246 & & $05 / 07 / 26$ & \\
\hline WFS72 & IRAS $19217+1651$ & 192358.6 & +165738 & & $05 / 07 / 27$ & \\
\hline WFS74 & IRAS $19266+1745$ & 192855.5 & +175200 & & $05 / 07 / 30$ & \\
\hline WFS75 & IRAS $19282+1814$ & 193023.1 & +182022 & & $05 / 07 / 30$ & \\
\hline WFS76 & IRAS $19282+1814$ & 193029.7 & +182037 & & $05 / 07 / 30$ & \\
\hline WFS77 & IRAS $19403+2258$ & 194228.8 & +230503 & & $05 / 07 / 28$ & 05/07/07 \\
\hline WFS78 & IRAS $19410+2336$ & 194310.6 & +234502 & $04 / 05 / 14$ & $04 / 05 / 14$ & 05/07/07 \\
\hline
\end{tabular}


Table 1. continued.

\begin{tabular}{|c|c|c|c|c|c|c|}
\hline \multirow[t]{2}{*}{ WFS } & \multirow[t]{2}{*}{ IRAS name } & \multirow{2}{*}{$\frac{\mathrm{RA}}{\mathrm{(J2000)}}$} & \multirow{2}{*}{$\begin{array}{c}\text { Dec } \\
(\mathrm{J} 2000)\end{array}$} & \multicolumn{3}{|c|}{ Dates of observations } \\
\hline & & & & $\mathrm{C}^{18} \mathrm{O}(2-1)$ & $\mathrm{C}^{17} \mathrm{O}(2-1)$ & $\mathrm{C}^{17} \mathrm{O}(3-2)$ \\
\hline WFS79 & IRAS $19410+2336$ & 194311.2 & +234406 & $04 / 05 / 14$ & $04 / 05 / 14,04 / 05 / 23$ & $05 / 07 / 07$ \\
\hline WFS80 & IRAS $19411+2306$ & 194317.6 & +231357 & & $05 / 07 / 28$ & 05/07/07 \\
\hline WFS81 & IRAS $19413+2332$ & 194326.3 & +234026 & & $05 / 07 / 28$ & 05/07/07 \\
\hline WFS82 & IRAS $19413+2332$ & 194329.0 & +234019 & & $05 / 07 / 29$ & 05/07/07 \\
\hline WFS83 & IRAS $19471+2641$ & 194910.1 & +264910 & & $05 / 07 / 29$ & 05/07/07 \\
\hline WFS84 & IRAS $19471+2641$ & 194911.8 & +264938 & & $05 / 07 / 29$ & 05/07/07 \\
\hline WFS85 & IRAS $20051+3435$ & 200704.5 & +344445 & & $04 / 05 / 23$ & \\
\hline WFS86 & IRAS $20081+2720$ & 201012.6 & +272913 & & $05 / 07 / 26$ & \\
\hline WFS87 & IRAS $20081+2720$ & 201013.3 & +272821 & & $04 / 05 / 23$ & \\
\hline WFS 88 & IRAS $20081+2720$ & 201016.0 & +272812 & & $04 / 05 / 23$ & \\
\hline WFS89 & IRAS $20081+2720$ & 201018.7 & +272718 & & $05 / 07 / 26$ & \\
\hline WFS90 & IRAS 20126+4104 & 201425.7 & +411330 & $04 / 05 / 14$ & $04 / 05 / 13$ & $05 / 07 / 21$ \\
\hline WFS91 & IRAS $20205+3948$ & 202220.0 & +395821 & $04 / 05 / 14$ & $04 / 05 / 13$ & $05 / 07 / 21$ \\
\hline WFS92 & IRAS 20205+3948 & 202224.9 & +395755 & & $05 / 07 / 26$ & \\
\hline WFS93 & IRAS $20216+4107$ & 202323.9 & +411742 & & $05 / 07 / 26$ & \\
\hline WFS94 & IRAS $20293+3952$ & 203112.9 & +400321 & & $05 / 07 / 27$ & \\
\hline WFS95 & IRAS 20319+3958 & 203349.4 & +400832 & & $04 / 05 / 15$ & \\
\hline WFS96 & IRAS $20332+4124$ & 203458.7 & +413446 & & $04 / 05 / 15$ & \\
\hline WFS97 & IRAS $20332+4124$ & 203501.1 & +413459 & & $04 / 05 / 15$ & \\
\hline WFS98 & IRAS $20343+4129$ & 203603.4 & +413944 & & $05 / 07 / 27$ & \\
\hline WFS99 & IRAS $20343+4129$ & 203606.3 & +413959 & $04 / 05 / 17$ & $04 / 05 / 23$ & \\
\hline WFS100 & IRAS $20343+4129$ & 203608.1 & +413958 & $04 / 05 / 17$ & $04 / 05 / 23$ & \\
\hline WFS101 & IRAS 22134+5834 & 221508.9 & +584908 & & $05 / 07 / 23$ & \\
\hline WFS 102 & IRAS $22551+6221$ & 225704.3 & +623744 & & $05 / 07 / 23$ & \\
\hline WFS103 & IRAS $22551+6221$ & 225707.4 & +623729 & & $05 / 07 / 23$ & \\
\hline WFS104 & IRAS $22551+6221$ & 225711.6 & +623646 & & $05 / 07 / 23$ & \\
\hline WFS107 & IRAS $22570+5912$ & 225905.0 & +592823 & $04 / 05 / 13$ & $04 / 05 / 13$ & $05 / 07 / 26$ \\
\hline WFS108 & IRAS $23033+5951$ & 230524.8 & +600814 & $04 / 05 / 13$ & $04 / 05 / 13$ & $05 / 07 / 26$ \\
\hline WFS109 & IRAS $23139+5939$ & 231609.8 & +595531 & $04 / 05 / 13$ & $04 / 05 / 13$ & $05 / 07 / 26$ \\
\hline WFS 110 & IRAS $23151+5912$ & 231720.4 & +592851 & $04 / 05 / 14$ & $04 / 05 / 22$ & $05 / 07 / 26$ \\
\hline WFS111 & IRAS $23545+6508$ & 235702.1 & +652438 & $04 / 05 / 14$ & $04 / 05 / 22$ & $05 / 07 / 26$ \\
\hline WFS112 & IRAS $23545+6508$ & 235706.4 & +652449 & $04 / 05 / 14$ & $04 / 05 / 22$ & $05 / 07 / 26$ \\
\hline
\end{tabular}

Table 2. Line widths and velocities for sources with two component $\mathrm{C}^{18} 0$ line profiles. All columns (except Col. 1) in units of km s${ }^{-1}$. The Gaussian linewidths were obtained using SPECX, whilst the hyperfine linewidths were obtained using the hfs method in CLASS.

\begin{tabular}{|c|c|c|c|c|c|c|c|c|c|c|}
\hline \multirow[b]{2}{*}{ WFS } & \multicolumn{4}{|c|}{$\mathrm{C}^{17} \mathrm{O} J=2 \rightarrow 1$} & \multicolumn{6}{|c|}{$\mathrm{C}^{18} \mathrm{O} J=2 \rightarrow 1$} \\
\hline & $\Delta v$ (Gauss) & $v_{\text {lsr }}$ & $\Delta v(\mathrm{hfs})$ & $v_{\text {lsr }}$ & $\Delta v$ (Gauss) & $v_{\mathrm{lsr}}$ & $\Delta v$ (narrow) & $v_{\text {lsr }}$ & $\Delta v$ (broad) & $v_{\text {lsr }}$ \\
\hline 14 & 2.84 & 33.26 & 2.44 & 33.22 & 2.42 & 33.26 & 2.14 & 33.21 & 3.31 & 33.47 \\
\hline 16 & 3.35 & 59.21 & 2.99 & 59.19 & 3.36 & 59.22 & 2.43 & 60.40 & 2.46 & 58.63 \\
\hline 17 & 3.02 & 45.12 & 2.65 & 45.09 & 2.72 & 45.08 & 2.20 & 44.87 & 3.70 & 45.67 \\
\hline 20 & 2.99 & 34.35 & 2.70 & 34.29 & 2.82 & 34.39 & 1.60 & 34.99 & 2.97 & 34.03 \\
\hline 21 & 2.76 & 34.40 & 2.25 & 34.35 & 2.25 & 34.39 & 1.97 & 34.46 & 3.94 & 33.61 \\
\hline 29 & 2.59 & 59.28 & 2.18 & 59.28 & 2.69 & 58.94 & 1.83 & 58.42 & 1.93 & 59.93 \\
\hline 30 & 3.05 & 95.64 & 2.96 & 95.75 & 2.44 & 95.69 & 1.46 & 95.65 & 5.80 & 95.79 \\
\hline 39 & 3.08 & 96.07 & 2.61 & 96.03 & 2.64 & 96.07 & 1.55 & 96.36 & 3.72 & 95.53 \\
\hline 78 & 2.82 & 21.84 & 2.47 & 21.80 & 2.83 & 21.72 & 0.84 & 21.70 & 3.10 & 21.73 \\
\hline 79 & 2.91 & 22.60 & 2.33 & 22.54 & 2.85 & 22.53 & 1.30 & 22.49 & 3.96 & 22.66 \\
\hline 90 & 3.25 & -3.63 & 2.94 & -3.67 & 2.83 & -3.64 & 1.28 & -3.89 & 3.59 & -3.41 \\
\hline 108 & 3.06 & -53.06 & 2.87 & -53.10 & 2.79 & -53.18 & 1.50 & -53.06 & 4.16 & -53.37 \\
\hline 109 & 2.56 & -44.35 & 2.33 & -44.38 & 2.74 & -44.29 & 1.80 & -44.58 & 3.57 & -43.82 \\
\hline 110 & 2.97 & -55.00 & 2.09 & -54.74 & 2.31 & -54.71 & 1.35 & -54.66 & 3.41 & -54.24 \\
\hline 112 & 2.03 & -18.53 & 1.55 & -18.62 & 1.84 & -18.45 & 1.18 & -17.41 & 1.31 & -18.65 \\
\hline
\end{tabular}


H. S. Thomas and G. A. Fuller: HMPOs: depletion, Online Material p 3

Table 4. Observed line parameters. The columns give the peak intensity, velocity, linewidth and integrated intensity for $\mathrm{C}^{17} \mathrm{O}$. The first three parameters for each transition are derived from hyperfine fitting of the data using CLASS. The integrated intensity was obtained using a single Gaussian fit. Three sigma upper limits are shown for sources where a line was not detected above this threshold. A * indicates data that has been observed in the previous year and is given in Table 3.

\begin{tabular}{|c|c|c|c|c|c|c|c|c|}
\hline \multirow[b]{2}{*}{ WFS } & \multicolumn{4}{|c|}{$\mathrm{C}^{17} \mathrm{O} J=2 \rightarrow 1$} & \multicolumn{4}{|c|}{$\mathrm{C}^{17} \mathrm{O} J=3 \rightarrow 2$} \\
\hline & $\begin{array}{l}\text { Peak } T_{\mathrm{mb}} \\
(\mathrm{K})\end{array}$ & $\begin{array}{c}v_{\text {lsr }} \\
\left(\mathrm{km} \mathrm{s}^{-1}\right)\end{array}$ & $\begin{array}{c}\Delta v \\
\left(\mathrm{~km} \mathrm{~s}^{-1}\right)\end{array}$ & $\int_{\left(\mathrm{K} \mathrm{km} \mathrm{s} ~^{-1}\right)} T_{\mathrm{mb}} \mathrm{d} v$ & $\begin{array}{c}\text { Peak } T_{\mathrm{mb}} \\
(\mathrm{K})\end{array}$ & $\begin{array}{c}v_{\text {lsr }} \\
\left(\mathrm{km} \mathrm{s}^{-1}\right)\end{array}$ & $\begin{array}{c}\Delta v \\
\left(\mathrm{~km} \mathrm{~s}^{-1}\right)\end{array}$ & $\begin{array}{c}\int T_{\mathrm{mb}} \mathrm{d} v \\
\left(\mathrm{~K} \mathrm{~km} \mathrm{~s}^{-1}\right)\end{array}$ \\
\hline 1 & 1.04 & -17.33 & 2.14 & 2.64 & 1.80 & -17.42 & 2.06 & 4.56 \\
\hline 3 & 1.17 & 0.10 & 1.72 & 3.01 & 1.52 & 0.09 & 1.89 & 3.78 \\
\hline 4 & 0.57 & 0.80 & 2.40 & 2.35 & $<0.54$ & - & - & - \\
\hline 6 & 1.42 & 5.76 & 1.81 & 3.48 & 1.55 & 5.79 & 1.76 & 3.51 \\
\hline 12 & 1.46 & 110.88 & 2.47 & 4.71 & - & - & - & - \\
\hline 13 & 0.72 & 21.81 & 3.36 & 2.71 & - & - & - & - \\
\hline 15 & 1.65 & 59.92 & 2.86 & 5.70 & - & - & - & - \\
\hline 18 & 1.55 & 120.91 & 3.01 & 5.36 & - & - & - & - \\
\hline 19 & 1.83 & 44.07 & 2.70 & 6.67 & - & - & - & - \\
\hline 22 & $*$ & $*$ & $*$ & $*$ & 1.67 & 84.65 & 2.08 & 3.92 \\
\hline 23 & 2.80 & 84.17 & 2.12 & 7.30 & - & - & - & - \\
\hline 24 & 0.84 & 76.48 & 2.72 & 2.72 & - & - & - & - \\
\hline 28 & 2.75 & 84.09 & 2.13 & 8.16 & - & - & - & - \\
\hline 29 & $*$ & $*$ & $*$ & $*$ & 2.61 & 59.22 & 1.67 & 7.37 \\
\hline 33 & 2.80 & 110.09 & 2.76 & 8.81 & - & - & - & - \\
\hline 35 & 0.81 & 26.14 & 3.77 & 3.35 & - & - & - & - \\
\hline 37 & 2.77 & 105.46 & 2.10 & 7.39 & - & - & - & - \\
\hline 38 & 0.91 & 111.16 & 1.55 & 1.84 & - & - & - & - \\
\hline 39 & $*$ & $*$ & $*$ & $*$ & 2.03 & 96.37 & 2.08 & 5.48 \\
\hline 41 & $<0.44$ & - & - & - & - & - & - & - \\
\hline 42 & 0.77 & 98.07 & 2.90 & 2.80 & - & - & - & - \\
\hline 51 & $*$ & $*$ & $*$ & $*$ & 1.25 & 84.52 & 1.25 & 2.49 \\
\hline 55 & 0.91 & 49.50 & 3.96 & 5.17 & - & - & - & - \\
\hline 57 & 0.71 & 83.47 & 1.99 & 1.84 & - & - & - & - \\
\hline 58 & 1.58 & 82.75 & 4.25 & 7.80 & - & - & - & - \\
\hline 59 & 1.22 & 76.42 & 2.56 & 3.87 & - & - & - & - \\
\hline 60 & $<0.46$ & - & - & - & - & - & - & - \\
\hline 61 & 1.99 & 76.87 & 2.72 & 6.55 & - & - & - & - \\
\hline 62 & $<0.36$ & - & - & - & - & - & - & - \\
\hline 63 & 0.12 & 50.64 & 1.42 & 0.86 & - & - & - & - \\
\hline 64 & 1.28 & 10.40 & 3.22 & 4.75 & - & - & - & - \\
\hline 66 & 2.41 & 66.13 & 3.05 & 9.04 & - & - & - & - \\
\hline 67 & 1.01 & 32.62 & 3.17 & 3.96 & - & - & - & - \\
\hline 68 & 1.28 & 55.09 & 2.38 & 4.14 & - & - & - & - \\
\hline 69 & 0.85 & 54.54 & 2.39 & 2.20 & - & - & - & - \\
\hline 70 & 0.91 & 6.54 & 1.82 & 2.55 & - & - & - & - \\
\hline 71 & 0.87 & 14.17 & 2.93 & 3.14 & - & - & - & - \\
\hline 72 & 1.07 & 3.77 & 3.94 & 4.86 & - & - & - & - \\
\hline 74 & 0.96 & 4.82 & 3.34 & 3.77 & - & - & - & - \\
\hline 75 & 2.93 & 23.09 & 1.45 & 5.97 & - & - & - & - \\
\hline 76 & 2.74 & 24.04 & 1.18 & 4.75 & - & - & - & - \\
\hline 77 & 2.81 & 26.66 & 1.05 & 4.20 & 5.03 & 26.72 & 0.89 & 6.14 \\
\hline 78 & $*$ & $*$ & $*$ & $*$ & 1.12 & 21.49 & 2.32 & 3.14 \\
\hline 79 & $*$ & $*$ & $*$ & $*$ & 1.93 & 22.45 & 2.26 & 5.86 \\
\hline 80 & 1.55 & 28.86 & 1.85 & 3.90 & 2.10 & 29.01 & 1.86 & 4.97 \\
\hline 81 & 1.99 & 20.29 & 1.69 & 4.38 & 1.17 & 20.43 & 0.94 & 2.63 \\
\hline 82 & 3.13 & 20.06 & 1.81 & 7.77 & 4.23 & 19.96 & 1.48 & 8.02 \\
\hline 83 & 0.94 & 21.47 & 1.66 & 2.43 & 0.97 & 21.60 & 1.40 & 1.95 \\
\hline 84 & 1.19 & 22.32 & 2.01 & 3.55 & 1.42 & 22.54 & 1.49 & 2.54 \\
\hline 86 & 0.87 & 5.72 & 1.94 & 2.55 & - & - & - & - \\
\hline 89 & 0.80 & 5.47 & 0.92 & 1.04 & - & - & - & - \\
\hline 90 & $*$ & $*$ & $*$ & $*$ & 1.49 & -3.48 & 3.10 & 6.52 \\
\hline 91 & $*$ & * & $*$ & $*$ & 4.96 & -1.89 & 1.10 & 7.81 \\
\hline 92 & 0.83 & -1.69 & 1.63 & 1.88 & - & - & - & - \\
\hline 93 & 1.48 & -1.69 & 1.60 & 3.57 & - & - & - & - \\
\hline 94 & 1.36 & 6.03 & 2.61 & 4.13 & - & - & - & - \\
\hline 98 & 1.83 & 11.50 & 1.03 & 2.90 & - & - & - & - \\
\hline 101 & 1.81 & -18.38 & 1.81 & 4.41 & - & - & - & - \\
\hline 102 & $<0.65$ & - & - & - & - & - & - & - \\
\hline 103 & $<0.69$ & - & - & - & - & - & - & - \\
\hline 104 & 1.52 & -12.35 & 0.95 & 2.38 & - & - & - & - \\
\hline 107 & $*$ & $*$ & $*$ & $*$ & 1.83 & -45.97 & 1.57 & 3.75 \\
\hline 108 & $*$ & $*$ & $*$ & $*$ & 1.51 & -53.21 & 2.70 & 5.03 \\
\hline 109 & $*$ & $*$ & $*$ & $*$ & 1.49 & -44.37 & 2.58 & 4.83 \\
\hline 110 & $*$ & $*$ & $*$ & $*$ & 1.80 & -54.75 & 2.00 & 4.67 \\
\hline 111 & $*$ & $*$ & $*$ & $*$ & 4.77 & -17.84 & 1.09 & 7.00 \\
\hline 112 & $*$ & $*$ & $*$ & $*$ & 3.13 & -18.59 & 1.24 & 4.95 \\
\hline
\end{tabular}


H. S. Thomas and G. A. Fuller: HMPOs: depletion, Online Material p 4

Table 5. Column densities calculated from the line intensities. The hydrogen column density corresponds to a $20^{\prime \prime}$ beam for direct comparison with the $J=2 \rightarrow 1$ observations.

\begin{tabular}{|c|c|c|c|c|c|c|c|c|c|}
\hline & $N\left(\mathrm{C}^{17} \mathrm{O}\right)$ & $N\left(\mathrm{C}^{17} \mathrm{O}\right)$ & $N\left(\mathrm{H}_{2}\right)$ & $\frac{N\left(\mathrm{C}^{17} \mathrm{O}_{2-1}\right)}{N\left(\mathrm{H}_{2}\right)}$ & & $N\left(\mathrm{C}^{17} \mathrm{O}\right)$ & $N\left(\mathrm{C}^{17} \mathrm{O}\right)$ & $N\left(\mathrm{H}_{2}\right)$ & $\frac{N\left(\mathrm{C}^{17} \mathrm{O}_{2-1}\right)}{N\left(\mathrm{H}_{2}\right)}$ \\
\hline WFS & $\begin{array}{c}J=2 \rightarrow 1 \\
\left(\times 10^{15}\right)\end{array}$ & $\begin{array}{c}J=3 \rightarrow 2 \\
\left(\times 10^{15}\right)\end{array}$ & $\left(\times 10^{22}\right)$ & $\left(\times 10^{-8}\right)$ & WFS & $\begin{array}{c}J=2 \rightarrow 1 \\
\left(\times 10^{15}\right)\end{array}$ & $\begin{array}{c}J=3 \rightarrow 2 \\
\left(\times 10^{15}\right)\end{array}$ & $\left(\times 10^{22}\right)$ & $\left(\times 10^{-8}\right)$ \\
\hline 1 & 0.64 & 0.90 & 4.03 & 1.58 & 68 & 1.00 & - & 2.86 & 3.50 \\
\hline 3 & 0.73 & 0.74 & 2.87 & 2.53 & 69 & 0.53 & - & - & - \\
\hline 4 & 0.57 & 0.00 & 2.38 & 2.38 & 70 & 0.62 & - & 1.90 & 3.24 \\
\hline 6 & 0.84 & 0.69 & 3.57 & 2.35 & 71 & 0.76 & - & 3.34 & 2.27 \\
\hline 12 & 1.14 & - & 4.16 & 2.73 & 72 & 1.21 & - & 9.64 & 1.25 \\
\hline 13 & 0.65 & - & 10.01 & 0.65 & 74 & 0.91 & - & 6.97 & 1.31 \\
\hline 14 & 1.87 & - & 8.15 & 2.30 & 75 & 1.44 & - & 5.96 & 2.42 \\
\hline 15 & 1.37 & - & 1.62 & 8.47 & 76 & 1.15 & - & 2.03 & 5.66 \\
\hline 16 & 3.39 & - & 12.09 & 2.80 & 77 & 1.01 & 1.21 & 2.11 & 4.81 \\
\hline 17 & 2.81 & - & 4.96 & 5.67 & 78 & 0.98 & 0.62 & 3.60 & 2.72 \\
\hline 18 & 1.29 & - & 6.09 & 2.13 & 79 & 1.33 & 1.15 & 12.15 & 1.10 \\
\hline 19 & 1.61 & - & 23.25 & 0.69 & 80 & 0.94 & 0.98 & 4.09 & 2.30 \\
\hline 20 & 1.01 & - & 0.98 & 10.28 & 81 & 1.06 & 0.52 & 1.80 & 5.87 \\
\hline 21 & 1.26 & - & 1.20 & 10.47 & 82 & 1.88 & 1.58 & 3.06 & 6.13 \\
\hline 22 & 1.93 & 0.77 & 5.53 & 3.50 & 83 & 0.59 & 0.38 & 1.54 & 3.81 \\
\hline 23 & 1.76 & - & - & - & 84 & 0.86 & 0.50 & 1.62 & 5.29 \\
\hline 24 & 0.66 & - & 2.50 & 2.63 & 85 & 1.07 & - & 2.53 & 4.25 \\
\hline 25 & 1.84 & - & 7.81 & 2.36 & 86 & 0.54 & - & 1.00 & 5.43 \\
\hline 28 & 1.97 & - & 4.31 & 4.57 & 87 & 1.22 & - & 2.73 & 4.47 \\
\hline 29 & 1.66 & 1.45 & 3.06 & 5.99 & 88 & 1.64 & - & 2.73 & 6.01 \\
\hline 30 & 0.89 & - & 4.44 & 2.01 & 89 & 0.25 & - & 0.91 & 2.77 \\
\hline 33 & 2.13 & - & 6.13 & 3.47 & 90 & 1.34 & 1.29 & 8.40 & 1.59 \\
\hline 34 & 2.65 & - & 4.31 & 6.15 & 91 & 1.40 & 1.54 & 2.53 & 5.52 \\
\hline 35 & 0.81 & - & 6.13 & 1.32 & 92 & 0.50 & - & 1.34 & 3.74 \\
\hline 36 & 1.54 & - & 3.24 & 4.76 & 93 & 0.86 & - & 2.91 & 2.96 \\
\hline 37 & 1.78 & - & 3.47 & 5.15 & 94 & 1.00 & - & 6.77 & 1.47 \\
\hline 38 & 0.43 & - & 2.33 & 1.85 & 95 & 0.68 & - & 1.15 & 5.88 \\
\hline 39 & 1.56 & 1.08 & 3.63 & 4.30 & 96 & 1.23 & - & 3.60 & 3.42 \\
\hline 41 & - & - & 0.08 & - & 97 & 1.06 & - & 2.86 & 3.71 \\
\hline 42 & 0.68 & - & 0.87 & 7.74 & 98 & 0.70 & - & - & - \\
\hline 51 & 1.25 & 0.49 & 1.36 & 9.17 & 99 & 1.02 & - & 5.28 & 1.93 \\
\hline 55 & 1.25 & - & 3.28 & 3.18 & 100 & 1.16 & - & - & - \\
\hline 57 & 0.44 & - & 2.66 & 1.67 & 101 & 1.06 & - & 3.29 & 3.24 \\
\hline 58 & 1.88 & - & 7.69 & 2.45 & 102 & - & - & - & - \\
\hline 59 & 0.93 & - & 3.86 & 2.42 & 103 & - & - & 2.31 & - \\
\hline 60 & - & - & 0.78 & - & 104 & 0.57 & - & 1.38 & 4.15 \\
\hline 61 & 1.58 & - & 5.82 & 2.72 & 107 & 0.73 & 0.74 & 2.88 & 2.53 \\
\hline 62 & 0.24 & - & 1.11 & 2.18 & 108 & 1.43 & 0.99 & 6.66 & 2.13 \\
\hline 63 & 0.21 & - & 0.47 & 4.35 & 109 & 0.97 & 0.95 & 7.75 & 1.25 \\
\hline 64 & 1.15 & - & 4.05 & 2.83 & 110 & 0.87 & 0.92 & 2.69 & 3.26 \\
\hline 66 & 2.18 & - & 2.86 & 3.83 & 111 & 1.51 & 1.38 & 2.67 & 5.68 \\
\hline 67 & 0.96 & - & 6.31 & 1.51 & 112 & 0.97 & 0.98 & 2.67 & 3.64 \\
\hline
\end{tabular}

